\title{
An efficient explicit full-discrete scheme for strong approximation of stochastic Allen-Cahn equation*
}

\author{
Xiaojie Wang \\ School of Mathematics and Statistics, \\ Central South University, Changsha, Hunan, China \\ e-mail: $\mathrm{x} \cdot \mathrm{j}$.wang7@csu.edu.cn
}

\begin{abstract}
In [Becker and Jentzen, Stoch. Proc. Appl. 129:28-69, 2019] and [Becker, et al., arXiv preprint arXiv:1711.02423, 2017], an explicit temporal semi-discretization scheme and a space-time full-discretization scheme were, respectively, introduced and analyzed for the additive noise-driven stochastic Allen-Cahn type equations, with strong convergence rates recovered. The present work aims to propose a different explicit full-discrete scheme to numerically solve the stochastic Allen-Cahn equation with cubic nonlinearity, perturbed by additive space-time white noise. The approximation is easily implementable, performing the spatial discretization by a spectral Galerkin method and the temporal discretization by a kind of nonlinearity-tamed accelerated exponential integrator scheme. Error bounds in a strong sense are analyzed for both the spatial semi-discretization and the spatio-temporal full discretization, with convergence rates in both space and time explicitly identified. It turns out that the obtained convergence rate of the new scheme is, in the temporal direction, twice as high as existing ones in the literature. Numerical results are finally reported to confirm the previous theoretical findings.
\end{abstract}

MSC 2010 subject classifications: $65 \mathrm{C} 30,60 \mathrm{H} 35,60 \mathrm{H} 15$.

Keywords and phrases: stochastic Allen-Cahn equation, cubic nonlinearity, spectral Galerkin method, tamed exponential integrator scheme, strong convergence rate.

\section{Introduction}

As an active area of research, numerical study of evolutionary stochastic partial differential equations (SPDEs) has attracted increasing attention in the past decades (see, e.g., monographs [35, 38, 31] and references therein). Albeit much progress has been made, it is still not well-understood, especially for numerical analysis of SPDEs with non-globally Lipschitz nonlinearities. The present work attempts to make a contribution in this direction and examine a space-time full-discretization scheme for a typical example of parabolic SPDEs with super-linearly growing nonlinearity, i.e., the stochastic Allen-Cahn equation. The driving noise is a space-time white one, which is of special interest as it can best model the fluctuations generated by microscopic effects in a homogeneous physical system, including, for example, molecular collisions in gases and liquids, electric fluctuations in resistors [18]. A lot of researchers carried out numerical analysis of SPDEs subject to such noise, e.g., $[36,16,19,21,13,30,4,5,26,1,15,14,28,8,37,9,42,49]$, to just mention a few.

Numerically solving the continuous problem on a computer forces us to perform both spatial and temporal discretizations. In space, we discretize SPDE (2.1) by a spectral Galerkin method, resulting in a system of finite dimensional stochastic differential equations (SDEs). Based on the spatial discretization, we propose a nonlinearity-tamed accelerated exponential time-stepping scheme given by (4.1). The resulting approximation errors of both the spatial discretization and the space-time fully discrete scheme are carefully analyzed, with strong convergence rates successfully recovered. More accurately, by $X\left(t_{m}\right)$ we denote the unique mild solution of the underlying SPDE taking values at temporal grid points $t_{m}=m \tau, m \in\{0,1, \ldots, M\}$ with uniform time step-size $\tau=\frac{T}{M}>0$ and by $Y_{t_{m}}^{M, N}$ the numerical

*This work was supported by NSF of China $(11671405,11971488,91630312)$, Natural Science Foundation of Hunan Province (2020JJ2040, 2018JJ3628). The author wants to thank the Tianyuan Mathematical Center in Northeast China for the hospitality when this work was presented in a conference in June of 2018 , hosted by the center. The author also wants to thank Yuying Zhao for her kind help with excellent typesetting and Ruisheng Qi and Meng Cai for their useful comments based on carefully reading the manuscript. 
approximations of $X\left(t_{m}\right)$, produced by the proposed fully discrete scheme. The approximation error measured in $L^{p}(\Omega ; H), p \in[2, \infty)$ reads (cf. Theorem 4.12):

$$
\sup _{0 \leq m \leq M}\left\|X\left(t_{m}\right)-Y_{t_{m}}^{M, N}\right\|_{L^{p}(\Omega ; H)} \leq C\left(N^{-\beta}+\tau^{\beta}\right), \quad \forall \beta \in\left(0, \frac{1}{2}\right) .
$$

Here $H:=L^{2}((0,1) ; \mathbb{R})$ and the constant $C$ depends on $p, \beta, T$ and the initial value of the SPDE, but does not depend on the discretization parameters $M, N$.

Over the last two years, several research works were reported on numerical analysis of space-time white noise driven SPDEs with cubic (polynomial) nonlinearity [4, 3, 37, 8, 6]. Becker and Jentzen [4] in 2016 introduced two nonlinearity-truncated Euler-type approximations for pure time discretizations of stochastic Ginzburg Landau type equations with slightly more general polynomials. There a strong convergence rate of order almost $\frac{1}{4}$ is identified. More recently when the first preprint of this work was almost finished, we were aware of four other preprints $[3,8,37,6]$ submitted to arXiv, concerning with numerical approximations of similar SPDEs. Becker, Gess, Jentzen and Kloeden [3] proposed new types of truncated exponential Euler space-time fully discrete schemes for the same problem as in [4] and derived strong convergence rates of order almost $\frac{1}{2}$ in space and order almost $\frac{1}{4}$ in time. Later, Bréhier and Goudenège [8] and Bréhier, Cui and Hong [6] analyzed some splitting time discretization schemes and obtained strong convergence rates of order $\frac{1}{4}$. Liu and Qiao [37] investigated a spectral Galerkin backward implicit Euler full discretization, with strong convergence rates of order almost $\frac{1}{2}$ in space and order $\frac{1}{4}$ in time achieved. As clearly implied by (1.1), the spatial convergence rate coincides with those in $[3,37]$, but the strong convergence rate of our time-stepping scheme can be of order almost $\frac{1}{2}$, essentially twice as high as those in $[4,3,37,8,6]$. Despite getting involved with linear functionals of the noise process, the newly proposed scheme is explicit, easily implementable and does not cost additional computational efforts (see comments in section 5 for the implementation of the linear functionals of the noise process).

It is important to emphasize that, proving the error estimate (1.1) is challenging, confronted with two essential difficulties, one being to derive uniform a priori moment bounds for the numerical approximations with super-linearly growing nonlinearity (see relevant comments in [3]) and the other to recover the temporal convergence rate of order almost $\frac{1}{2}$, instead of order (almost) $\frac{1}{4}$ in the existing literature. With regard to the former, we essentially rely on certain estimates for deterministic perturbed PDEs (4.3), as elaborated in subsection 4. The uniform a priori moment $L_{\infty}$-bounds are derived based on a certain bootstrap argument, by showing $\mathbb{E}\left[\mathbb{1}_{\Omega_{R^{\tau}, t_{m}}}\left\|Y_{t_{m}}^{M, N}\right\|_{V}^{p}\right]<\infty$ and $\mathbb{E}\left[\mathbb{1}_{\Omega_{R^{\tau}, t_{m}}^{c}}\left\|Y_{t_{m}}^{M, N}\right\|_{V}^{p}\right]<\infty$, $V:=C((0,1), \mathbb{R})$, for subevents $\Omega_{R^{\tau}, t_{m}}$ with $R^{\tau}$ depending on $\tau$ carefully chosen (see subsection 4.2). The latter difficulty is addressed by fully exploiting the global monotonicity condition on the nonlinearity in conjunction with smoothing property of the analytic semigroup, commutativity properties of the nonlinearity and improved temporal Hölder regularity results in negative Sobolev spaces (consult subsection 4.3 and particularly the treatment of $J_{1}$ in the proof of Theorem 4.12 for details).

Furthermore, we would like to point out that the improvement of convergence rate is essentially credited to fully preserving the stochastic convolution in the time-stepping scheme (4.1). Such a kind of accelerating technique was used by Jentzen and Kloeden [30], to solve nearly linear parabolic SPDEs and has been further examined and extended in different settings [29, 50, 49, 43, 39], where a globally Lipschitz condition imposed on nonlinearity is indispensable in the error analysis. When the nonlinearity grows super-linearly and the globally Lipschitz condition is thus violated, one can in general not expect the usual accelerated exponential time-stepping schemes converge in the strong sense, based on the observations in [2,24]. To address this issue, we introduce a taming technique previously used in $[25,48,47,23,27]$ for ordinary SDEs, and propose a nonlinearity-tamed version of accelerated exponential Euler scheme for the time discretization. Although the idea of constructing the explicit scheme in this paper is inspired by the aforementioned works, the approach of the error analysis for the full discretization of the stochastic Allen-Cahn equation with space-time white noise is original and much more involved than that in both the finite dimensional non-globally Lipschitz SDE setting and the globally Lipschitz SPDE setting (see section 4). Moreover, our approach is much easier than that in [3], also 
treating explicit full-discrete schemes for Allen-Cahn type SPDEs.

Finally, we mention that, just one spatial dimension is considered here because the space-time white noise driven SPDE only allows for a mild solution with a positive (but very low) order of regularity in one spatial dimension. It is because of the low order of regularity that the error analysis becomes difficult. Further, the error analysis of the space-time full discretization is significantly more involved than that of the pure time semi-discretization (compare [4] and [3] and see comments therein). Strong convergence analysis of numerical methods for smoother noise (e.g., trace-class noise) driven stochastic Allen-Cahn equation in multiple spatial dimensions has been done in our recent work [44] and further strong and weak approximations of parabolic SPDEs with non-globally Lipschitz nonlinearity will be our forthcoming works (see also, e.g., [7, 45, 33, 34, 40, 17, 20, 32, 22] for revalent topics).

The rest of this paper is organized as follows. In the next section we collect some basic facts and present the well-posedness of the stochastic problem under given assumptions. Section 3 and Section 4 are, respectively, devoted to the analysis of strong convergence rates for both the spatial semidiscretization and the spatio-temporal full discretization of the underlying SPDEs. Numerical results are included in section 5 to test previous theoretical findings.

\section{Well-posedness of the stochastic problem}

Throughout this article, we are interested in the additive space-time white noise driven stochastic AllenCahn equation with cubic nonlinearity, described by

$$
\left\{\begin{array}{l}
\frac{\partial u}{\partial t}(t, x)=\frac{\partial^{2} u}{\partial x^{2}}(t, x)+f(u(t, x))+\dot{W}(t, x), x \in D, t \in(0, T] \\
u(0, x)=u_{0}(x), x \in D \\
u(t, 0)=u(t, 1)=0, t \in(0, T] .
\end{array}\right.
$$

Here $D:=(0,1), T>0, f: \mathbb{R} \rightarrow \mathbb{R}$ is given by $f(v)=a_{3} v^{3}+a_{2} v^{2}+a_{1} v+a_{0}, a_{3}<0, a_{2}, a_{1}, a_{0}, v \in \mathbb{R}$, and $\dot{W}(t, \cdot)$ stands for a formal time derivative of a cylindrical I-Wiener process [12]. In order to define a mild solution of (2.1) following the semigroup approach in [12], we attempt to put everything into an abstract framework. Given a real separable Hilbert space $(H,\langle\cdot, \cdot\rangle,\|\cdot\|)$ with $\|\cdot\|=\langle\cdot, \cdot\rangle^{\frac{1}{2}}$, by $\mathscr{L}(H)$ we denote the space of bounded linear operators from $H$ to $H$ endowed with the usual operator norm $\|\cdot\|_{\mathscr{L}(H)}$. Additionally, we denote by $\mathscr{L}_{2}(H) \subset \mathscr{L}(H)$ the subspace consisting of all Hilbert-Schmidt operators from $H$ to $H$ [12]. It is known that $\mathscr{L}_{2}(H)$ is a separable Hilbert space, equipped with the scalar product $\left\langle\Gamma_{1}, \Gamma_{2}\right\rangle_{\mathscr{L}_{2}(H)}:=\sum_{n \in \mathbb{N}}\left\langle\Gamma_{1} \eta_{n}, \Gamma_{2} \eta_{n}\right\rangle$, and norm $\|\Gamma\|_{\mathscr{L}_{2}(H)}:=\left(\sum_{n \in \mathbb{N}}\left\|\Gamma \eta_{n}\right\|^{2}\right)^{\frac{1}{2}}$, independent of the particular choice of orthogonal basis $\left\{\eta_{n}\right\}_{n \in \mathbb{N}}$ of $H$. Below we sometimes write $\mathscr{L}_{2}:=\mathscr{L}_{2}(H)$ for brevity. If $\Gamma \in \mathscr{L}(H)$ and $\Gamma_{1}, \Gamma_{2} \in \mathscr{L}_{2}(H)$, then $\left|\left\langle\Gamma_{1}, \Gamma_{2}\right\rangle_{\mathscr{L}_{2}(H)}\right| \leq\left\|\Gamma_{1}\right\|_{\mathscr{L}_{2}(H)}\left\|\Gamma_{2}\right\|_{\mathscr{L}_{2}(H)},\left\|\Gamma \Gamma_{1}\right\|_{\mathscr{L}_{2}(H)} \leq$ $\|\Gamma\|_{\mathscr{L}(H)}\left\|\Gamma_{1}\right\|_{\mathscr{L}_{2}(H)}$. By $L^{\gamma}(D ; \mathbb{R}), \gamma \geq 1\left(L^{\gamma}(D)\right.$ for short) we denote a Banach space consisting of $\gamma-$ times integrable functions and by $V:=C(D, \mathbb{R})$ a Banach space of continuous functions with usual norms. To reformulate (2.1) as an abstract problem, we make the following assumptions.

Assumption 2.1 (Linear operator $A)$. Denote $D:=(0,1)$ and let $H=L^{2}(D ; \mathbb{R})$ be a real separable Hilbert space, equipped with usual product $\langle\cdot, \cdot\rangle$ and norm $\|\cdot\|=\langle\cdot, \cdot\rangle^{\frac{1}{2}}$. Let $-A: \operatorname{dom}(A) \subset H \rightarrow H$ be the Laplacian with homogeneous Dirichlet boundary conditions, defined by $-A u=\Delta u, u \in \operatorname{dom}(A):=$ $H^{2} \cap H_{0}^{1}$.

The above setting assures that there exists an increasing sequence of real numbers $\lambda_{i}=\pi^{2} i^{2}, i \in \mathbb{N}$ and an orthonormal basis $\left\{e_{i}(x)=\sqrt{2} \sin (i \pi x), x \in(0,1)\right\}_{i \in \mathbb{N}}$ such that $A e_{i}=\lambda_{i} e_{i}$. In particular, the linear unbounded operator $A$ is positive, i.e., $\langle-A v, v\rangle \leq-\lambda_{1}\|v\|^{2}$, for all $v \in \operatorname{dom}(A)$. Moreover, $-A$ generates an analytic semigroup $E(t)=e^{-t A}, t \geq 0$ on $H$ and we can define the fractional powers of $A$, i.e., $A^{\gamma}, \gamma \in \mathbb{R}$ and the Hilbert space $\dot{H}^{\gamma}:=\operatorname{dom}\left(A^{\frac{\gamma}{2}}\right)$, equipped with inner product $\langle\cdot, \cdot\rangle_{\gamma}:=\left\langle A^{\frac{\gamma}{2}} \cdot, A^{\frac{\gamma}{2}} \cdot\right\rangle$ and norm $\|\cdot\|_{\gamma}=\langle\cdot, \cdot\rangle_{\gamma}^{\frac{1}{2}}\left[35\right.$, Appendix B.2]. Moreover, $\dot{H}^{0}=H$ and $\dot{H}^{\gamma} \subset \dot{H}^{\delta}, \gamma \geq \delta$. It is well-known 
that [41], for a positive constant $c>0$,

$$
\begin{gathered}
\left\|A^{\gamma} E(t)\right\|_{\mathscr{L}(H)} \leq c t^{-\gamma}, \quad t>0, \gamma \geq 0, \\
\left\|A^{-\rho}(I-E(t))\right\|_{\mathscr{L}(H)} \leq c t^{\rho}, \quad t>0, \rho \in[0,1],
\end{gathered}
$$

and accordingly one can verify that [35, Lemma B.9 (iii)]

$$
\int_{s}^{t}\left\|A^{\frac{\rho}{2}} E(t-r) u\right\|^{2} \mathrm{~d} r \leq c(t-s)^{1-\rho}\|u\|^{2}, \quad \forall u \in H, \rho \in[0,1], 0 \leq s \leq t .
$$

Here the constant $c$, might depending on $\gamma$, can be chosen to be independent of $\rho \in[0,1]$. Also, it is evident to see that

$$
\left\|A^{\frac{\beta-1}{2}}\right\|_{\mathscr{L}_{2}(H)}=\pi^{\beta-1}\left(\sum_{i \in \mathbb{N}} i^{2(\beta-1)}\right)^{\frac{1}{2}}<\infty, \quad \text { for any } \beta<\frac{1}{2}
$$

Assumption 2.2 (Nonlinearity). Let $F: L^{6}(D ; \mathbb{R}) \rightarrow H$ be a deterministic mapping defined by

$F(v)(x)=f(v(x)):=a_{3} v^{3}(x)+a_{2} v^{2}(x)+a_{1} v(x)+a_{0}, x \in(0,1), a_{3}<0, a_{2}, a_{1}, a_{0} \in \mathbb{R}, v \in L^{6}(D ; \mathbb{R})$.

It is easy to find constants $L_{0}, L_{1} \in(0, \infty)$ such that

$$
\begin{aligned}
\langle u-v, F(u)-F(v)\rangle & \leq L_{0}\|u-v\|^{2}, \quad u, v \in V, \\
\|F(u)-F(v)\| & \leq L_{1}\left(1+\|u\|_{V}^{2}+\|v\|_{V}^{2}\right)\|u-v\|, \quad u, v \in V .
\end{aligned}
$$

The second property in (2.5) immediately implies, for some $L_{2} \in(0, \infty)$,

$$
\|F(u)\| \leq L_{1}\left(1+\|u\|_{V}^{2}\right)\|u\|+\|F(0)\| \leq L_{2}\left(1+\|u\|_{V}^{3}\right), \quad u \in V
$$

Evidently, the constants $L_{0}, L_{1}, L_{2}$ only depend on coefficients of $f$, i.e., $a_{0}, a_{1}, a_{2}, a_{3}$.

Assumption 2.3 (Noise process). Let $\{W(t)\}_{t \in[0, T]}$ be a cylindrical I-Wiener process on a probability space $(\Omega, \mathscr{F}, \mathbb{P})$ with a normal filtration $\left\{\mathscr{F}_{t}\right\}_{t \in[0, T]}$, represented by a formal series,

$$
W(t):=\sum_{n=1}^{\infty} \beta_{n}(t) e_{n}, \quad t \in[0, T]
$$

where $\left\{\beta_{n}(t)\right\}_{n \in \mathbb{N}}, t \in[0, T]$ is a sequence of independent real-valued standard Brownian motions and $\left\{e_{n}=\sqrt{2} \sin (n \pi x), x \in(0,1)\right\}_{n \in \mathbb{N}}$ is a complete orthonormal basis of $H$.

Assumption 2.4 (Initial value). Let the initial data $X_{0}: \Omega \rightarrow H$, given by $X_{0}(\cdot)=u_{0}(\cdot)$, be an $\mathscr{F} 0 / \mathscr{B}(H)$ measurable random variable. For sufficiently large positive number $p_{0} \in \mathbb{N}$ and for any $\beta<\frac{1}{2}$, there exists constants $K_{\beta}$ depending on $\beta, p_{0}$ and $\hat{K}_{V}$ only depending on $p_{0}$ such that

$$
\left\|X_{0}\right\|_{L^{p_{0}\left(\Omega, \dot{H}^{\beta}\right)}} \leq K_{\beta}<\infty, \quad\left\|X_{0}\right\|_{L^{p_{0}}(\Omega, V)} \leq \hat{K}_{V}<\infty
$$

At the moment, we are prepared to formulate the concrete problem (2.1) as an abstract stochastic evolution equation in the Hilbert space $H$,

$$
\left\{\begin{array}{l}
\mathrm{d} X(t)+A X(t) \mathrm{d} t=F(X(t)) \mathrm{d} t+\mathrm{d} W(t), \quad t \in(0, T] \\
X(0)=X_{0}
\end{array}\right.
$$

where $X(t, \cdot)=u(t, \cdot)$ and the abstract items $A, F, X_{0}$ are defined in Assumptions 2.1-2.4. The above assumptions suffice to establish the well-posedness and regularity results of the mild solution to (2.9), defined by (2.13) later. Before that, we provide some regularity properties of the stochastic convolution. 
Lemma 2.5. Let Assumptions 2.1, 2.3 be fulfilled. For any $p \in[2, \infty)$ there exists a constant $C_{0}$ dependent of $p$ but independent of $\beta \in\left[0, \frac{1}{2}\right)$ such that the stochastic convolution $\left\{\mathscr{O}_{t}\right\}_{t \in[0, T]}$ satisfies

$$
\begin{gathered}
\left\|\mathscr{O}_{t}\right\|_{L^{p}\left(\Omega, \dot{H}^{\beta}\right)} \leq C_{0}\left\|A^{\frac{\beta-1}{2}}\right\|_{\mathscr{L}_{2}(H)}<\infty, \quad \text { with } \quad \mathscr{O}_{t}:=\int_{0}^{t} E(t-s) d W(s), \\
\left\|\mathscr{O}_{t}-\mathscr{O}_{s}\right\|_{L^{p}(\Omega, H)} \leq C_{0}\left\|A^{\frac{\beta-1}{2}}\right\|_{\mathscr{L}_{2}(H)}(t-s)^{\frac{\beta}{2}}, \quad 0 \leq s<t \leq T .
\end{gathered}
$$

Moreover, we have

$$
\mathbb{E}\left[\sup _{t \in[0, T]}\left\|\mathscr{O}_{t}\right\|_{V}^{p}\right]<\infty .
$$

Recalling (2.3) one can validate the first two estimates easily, see, e.g., [50, Theorem 2.4.]. The last assertion can, e.g., be found in [11, Proposition 4.3] and [10, Lemma 6.1.2]. Owing to the above regularity properties of the stochastic convolution, we can get the corresponding regularity properties of the mild solution to (2.9) as follows.

Theorem 2.6. Under Assumptions 2.1-2.4, SPDE (2.9) possesses a unique mild solution $X:[0, T] \times$ $\Omega \rightarrow V$ with continuous sample paths, determined by,

$$
X(t)=E(t) X_{0}+\int_{0}^{t} E(t-s) F(X(s)) d s+\mathscr{O}_{t} \quad \mathbb{P} \text {-a.s. }
$$

For any $p \in[2, \infty)$ and $\beta<\frac{1}{2}$, there exists positive constants $C_{1}, C_{2}$ depending on $p, T,\left\{a_{i}\right\}_{i=0}^{3}$ but not depending on $\beta$ such that,

$$
\begin{aligned}
& \sup _{t \in[0, T]}\|X(t)\|_{L^{p}(\Omega, V)} \leq C_{1}\left(1+\left\|X_{0}\right\|_{L^{p}(\Omega, V)}\right), \\
& \sup _{t \in[0, T]}\|X(t)\|_{L^{p}\left(\Omega, \dot{H}^{\beta}\right)} \leq C_{2}\left(1+\left\|X_{0}\right\|_{L^{p}\left(\Omega, \dot{H}^{\beta}\right)}+\left\|X_{0}\right\|_{L^{3 p}(\Omega, V)}^{3}+\left\|A^{\frac{\beta-1}{2}}\right\|_{\mathscr{L}_{2}(H)}\right) .
\end{aligned}
$$

Moreover, there exists a constant $C_{3} \in[0, \infty)$ depending on $p, T,\left\{a_{i}\right\}_{i=0}^{3}$ but not depending on $\beta$ such that, for any $\beta<\frac{1}{2}$ and $0 \leq s<t \leq T$,

$$
\|X(t)-X(s)\|_{L^{p}(\Omega, H)} \leq C_{3}\left(1+\left\|X_{0}\right\|_{L^{p}\left(\Omega, \dot{H}^{\beta}\right)}+\left\|X_{0}\right\|_{L^{3 p}(\Omega, V)}^{3}+\left\|A^{\frac{\beta-1}{2}}\right\|_{\mathscr{L}_{2}(H)}\right)(t-s)^{\frac{\beta}{2}} .
$$

The existence of the unique mild solution and the regularity assertion (2.14) are based on [10, Proposition 6.2.2] and (2.12). The rest of estimates in Theorem 2.6 can be verified by standard arguments (consult, e.g., [50, Theorem 2.4.]).

\section{Spatial semi-discretization}

This section concerns the error analysis for a spectral Galerkin spatial semi-discretization of the underlying problem (2.9). For $N \in \mathbb{N}$ we define a finite dimensional subspace of $H$ by

$$
H^{N}:=\operatorname{span}\left\{e_{1}, e_{2}, \cdots, e_{N}\right\},
$$

and the projection operator $P_{N}: \dot{H}^{\alpha} \rightarrow H^{N}$ by $P_{N} \xi=\sum_{i=1}^{N}\left\langle\xi, e_{i}\right\rangle e_{i}, \forall \xi \in \dot{H}^{\alpha}, \alpha \in \mathbb{R}$. Here $H^{N}$ is chosen as the linear space spanned by the $N$ first eigenvectors of the dominant linear operator $A$. It is not difficult to deduce that

$$
\left\|\left(P_{N}-I\right) \varphi\right\| \leq \lambda_{N+1}^{-\frac{\alpha}{2}}\|\varphi\|_{\alpha} \leq N^{-\alpha}\|\varphi\|_{\alpha}, \quad \forall \varphi \in \dot{H}^{\alpha}, \alpha \geq 0 .
$$

Additionally, define $A_{N}: H \rightarrow H^{N}$ as $A_{N}=A P_{N}$, which generates an analytic semigroup $E_{N}(t)=e^{-t A_{N}}$, $t \in[0, \infty)$ in $H^{N}$. Then the spectral Galerkin approximation of (2.9) results in the following finite dimensional SDEs,

$$
\left\{\begin{array}{l}
\mathrm{d} X^{N}(t)+A_{N} X^{N}(t) \mathrm{d} t=F_{N}\left(X^{N}(t)\right) \mathrm{d} t+P_{N} \mathrm{~d} W(t), \quad t \in(0, T] \\
X^{N}(0)=P_{N} X_{0}
\end{array}\right.
$$


where we write $F_{N}:=P_{N} F$ for short. It is clear to see that (3.3) admits a unique solution in $H^{N}$. By the variation of constant, the corresponding solution can be written as

$$
X^{N}(t)=E_{N}(t) P_{N} X_{0}+\int_{0}^{t} E_{N}(t-s) P_{N} F\left(X^{N}(s)\right) \mathrm{d} s+\int_{0}^{t} E_{N}(t-s) P_{N} \mathrm{~d} W(s), \mathbb{P} \text {-a.s.. }
$$

In the error analysis for the spatial semi-discretization (3.3), we require $\left\|P_{N} X(t)\right\|_{L^{p}(\Omega, V)}, t>0$ to be uniformly bounded with respect to $N \in \mathbb{N}$, which can be deduced based on the following two auxiliary results. The first one is a direct consequence of [5, Lemma 5.4] with $t_{1}=0$ and we follow the same arguments there to provide a short proof here.

Lemma 3.1. Let $\left\{\mathscr{O}_{t}\right\}_{t \in[0, T]}$ be the stochastic convolution defined by (2.12) and let Assumptions 2.1, 2.3 be fulfilled. Then for any $p \in[2, \infty)$ it holds

$$
\sup _{t \in[0, T], N \in \mathbb{N}}\left\|\mathscr{O}_{t}^{N}\right\|_{L^{p}(\Omega, V)}<\infty, \quad \text { with } \mathscr{O}_{t}^{N}:=P_{N} \mathscr{O}_{t} .
$$

Proof of Lemma 3.1. Owing to Assumptions 2.1, 2.3, we perform the expansion of the stochastic convolution $O_{t}^{N}$ and use the Itô isometry to get, for any $N \in \mathbb{N}, t \in[0, T], x, y \in D$,

$$
\begin{aligned}
\mathbb{E}\left[\left|\mathscr{O}_{t}^{N}(x)-\mathscr{O}_{t}^{N}(y)\right|^{2}\right] & \leq \sum_{1 \leq i \leq N} \mathbb{E}\left[\left|\int_{0}^{t} e^{-\lambda_{i}(t-s)} \mathrm{d} \beta_{i}(s)\right|^{2}\right]\left|e_{i}(x)-e_{i}(y)\right|^{2} \\
& \leq \sum_{i \in \mathbb{N}}\left(2 \lambda_{i}\right)^{-1}(\sqrt{2} \pi i)^{\frac{4}{5}}|x-y|^{\frac{4}{5}}\left(\left|e_{i}(x)\right|+\left|e_{i}(y)\right|\right)^{\frac{6}{5}} \\
& \leq 2^{\frac{6}{5}} \pi^{-\frac{6}{5}}|x-y|^{\frac{4}{5}} \sum_{i \in \mathbb{N}} i^{-\frac{6}{5}} \\
& \leq 3|x-y|^{\frac{4}{5}}
\end{aligned}
$$

In the same manner, one can acquire, for any $N \in \mathbb{N}, t \in[0, T]$,

$$
\sup _{x \in D} \mathbb{E}\left[\left|\mathscr{O}_{t}^{N}(x)\right|^{2}\right] \leq 2
$$

Using the Sobolev embedding inequality $W^{\frac{1}{5}, p} \subset V, p>5$ and noting that the stochastic convolution $O_{t}^{N}$ is Gaussian one can deduce, for any $N \in \mathbb{N}, t \in[0, T]$,

$$
\begin{aligned}
\mathbb{E}\left[\left\|\mathscr{O}_{t}^{N}\right\|_{V}^{p}\right] & \leq C \int_{0}^{1} \mathbb{E}\left[\left|\mathscr{O}_{t}^{N}(x)\right|^{p}\right] \mathrm{d} x+C \int_{0}^{1} \int_{0}^{1} \frac{\mathbb{E}\left[\left|\mathscr{O}_{t}^{N}(x)-\mathscr{O}_{t}^{N}(y)\right|^{p}\right]}{|x-y|^{\frac{p}{5}+1}} \mathrm{~d} x \mathrm{~d} y \\
& \leq C \int_{0}^{1}\left(\mathbb{E}\left[\left|\mathscr{O}_{t}^{N}(x)\right|^{2}\right]\right)^{\frac{p}{2}} \mathrm{~d} x+C \int_{0}^{1} \int_{0}^{1} \frac{\left(\mathbb{E}\left[\left|\mathscr{O}_{t}^{N}(x)-\mathscr{O}_{t}^{N}(y)\right|^{2}\right]\right)^{\frac{p}{2}}}{|x-y|^{\frac{p}{5}+1}} \mathrm{~d} x \mathrm{~d} y \\
& \leq C\left(1+\int_{0}^{1} \int_{0}^{1}|x-y|^{\frac{p}{5}-1} \mathrm{~d} x \mathrm{~d} y\right)<\infty .
\end{aligned}
$$

Using Hölder's inequality yields the desired assertion in the case $p \in[2,5]$.

The second one concerns the smoothing property of the analytic semigroup.

Lemma 3.2. Let Assumptions 2.1 be fulfilled. For any $N \in \mathbb{N}$ and $\psi \in \dot{H}^{\gamma}, \gamma \in\left[0, \frac{1}{2}\right)$, it holds that

$$
\left\|P_{N} E(t) \psi\right\|_{V} \leq 2^{\gamma}\left(\frac{5-4 \gamma}{2 \pi(1-2 \gamma)}\right)^{\frac{1}{2}} t^{\frac{2 \gamma-1}{4}}\|\psi\|_{\gamma}, \quad t>0, \gamma \in\left[0, \frac{1}{2}\right)
$$


Proof of Lemma 3.2. Elementary facts readily yield

$$
\begin{aligned}
\left\|P_{N} E(t) \psi\right\|_{V} & =\sup _{x \in[0,1]}\left|\sum_{i=1}^{N} e^{-\lambda_{i} t}\left\langle\psi, e_{i}\right\rangle e_{i}(x)\right| \leq \sqrt{2} \sum_{i=1}^{N} e^{-\lambda_{i} t}\left|\left\langle\psi, e_{i}\right\rangle\right| \\
& \leq \sqrt{2}\left(\sum_{i=1}^{N} \lambda_{i}^{-\gamma} e^{-2 \lambda_{i} t}\right)^{1 / 2}\left(\sum_{i=1}^{N} \lambda_{i}^{\gamma}\left|\left\langle\psi, e_{i}\right\rangle\right|^{2}\right)^{1 / 2} \\
& \leq \sqrt{2} \pi^{-\gamma}\left(\int_{0}^{\infty} x^{-2 \gamma} e^{-2 \pi^{2} x^{2} t} \mathrm{~d} x\right)^{1 / 2}\|\psi\|_{\gamma} \\
& =2^{\gamma} \pi^{-\frac{1}{2} t^{\frac{2 \gamma-1}{4}}}\left(\int_{0}^{\infty} y^{-2 \gamma} e^{-y^{2} / 2} \mathrm{~d} y\right)^{1 / 2}\|\psi\|_{\gamma} \\
& \leq 2^{\gamma}\left(\frac{5-4 \gamma}{2 \pi(1-2 \gamma)}\right)^{\frac{1}{2}} t^{\frac{2 \gamma-1}{4}}\|\psi\|_{\gamma},
\end{aligned}
$$

as required.

Equipped with the above two estimates, one can prove that $\left\|P_{N} X(t)\right\|_{L^{p}(\Omega, V)}, t>0$ is uniformly bounded with respect to $N \in \mathbb{N}$ as follows.

Lemma 3.3. Let $\{X(t)\}_{t \in[0, T]}$ be the mild solution to (2.9), defined by (2.13). Then for any $p \in[2, \infty)$ and $\gamma \in\left[0, \frac{1}{2}\right)$ there exists a positive constant $C$, depending on $T, p,\left\{a_{i}\right\}_{i=0}^{3}$, but not depending on $N, \beta, X_{0}$ such that

$$
\sup _{N \in \mathbb{N}}\left\|P_{N} X(t)\right\|_{L^{p}(\Omega, V)} \leq 2^{\gamma}\left(\frac{5-4 \gamma}{2 \pi(1-2 \gamma)}\right)^{\frac{1}{2}}\left\|X_{0}\right\|_{L^{p}\left(\Omega, \dot{H}^{\gamma}\right)} t^{\frac{2 \gamma-1}{4}}+C\left(1+\left\|X_{0}\right\|_{L^{3 p}(\Omega, V)}^{3}\right), \quad t \in(0, T] .
$$

Proof of Lemma 3.3. Observing that $E_{N}(t) P_{N}=E(t) P_{N}$ and using Lemmas 3.1, 3.2 show

$$
\begin{aligned}
& \left\|P_{N} X(t)\right\|_{L^{p}(\Omega, V)} \leq\left\|E(t) P_{N} X_{0}\right\|_{L^{p}(\Omega, V)}+\int_{0}^{t}\left\|E(t-s) F_{N}(X(s))\right\|_{L^{p}(\Omega, V)} \mathrm{d} s+\left\|P_{N} \mathscr{O}_{t}\right\|_{L^{p}(\Omega, V)} \\
& \quad \leq 2^{\gamma}\left(\frac{5-4 \gamma}{2 \pi(1-2 \gamma)}\right)^{\frac{1}{2}} t^{\frac{2 \gamma-1}{4}}\left\|X_{0}\right\|_{L^{p}\left(\Omega, \dot{H}^{\gamma}\right)}+\int_{0}^{t}(t-s)^{-\frac{1}{4}}\|F(X(s))\|_{L^{p}(\Omega, H)} \mathrm{d} s+\left\|P_{N} \mathscr{O}_{t}\right\|_{L^{p}(\Omega, V)} \\
& \quad \leq 2^{\gamma}\left(\frac{5-4 \gamma}{2 \pi(1-2 \gamma)}\right)^{\frac{1}{2}} t^{\frac{2 \gamma-1}{4}}\left\|X_{0}\right\|_{L^{p}\left(\Omega, \dot{H}^{\gamma}\right)}+\frac{4}{3} t^{\frac{3}{4}} \sup _{s \in[0, T]}\|F(X(s))\|_{L^{p}(\Omega, H)}+\left\|P_{N} \mathscr{O}_{t}\right\|_{L^{p}(\Omega, V)} \\
& \quad \leq 2^{\gamma}\left(\frac{5-4 \gamma}{2 \pi(1-2 \gamma)}\right)^{\frac{1}{2}} t^{\frac{2 \gamma-1}{4}}\left\|X_{0}\right\|_{L^{p}\left(\Omega, \dot{H}^{\gamma}\right)}+C\left(1+\sup _{s \in[0, T]}\|X(s)\|_{L^{3 p}(\Omega, V)}^{3}\right)+\left\|P_{N} \mathscr{O}_{t}\right\|_{L^{p}(\Omega, V)} .
\end{aligned}
$$

Owing to (2.14), (3.5) and Assumption 2.4, one can arrive at the expected estimate.

Throughout this paper, by $C$ we mean deterministic constants, not necessarily the same at each occurrence, but independent of the discretization parameters. Now we are prepared to carry out convergence analysis for the spectral Galerkin discretization (3.3).

Theorem 3.4 (Spatial error estimate). Let Assumptions 2.1-2.4 hold. Let $X(t)$ and $X^{N}(t)$ be defined through (2.9) and (3.4), respectively. Then for any $\beta<\frac{1}{2}, p \in[2, \infty)$ and $N \in \mathbb{N}$, there exists a positive constant $C$, depending on $T, p,\left\{a_{i}\right\}_{i=0}^{3}$, but not depending on $N, \beta, X_{0}$ such that

$$
\sup _{t \in[0, T]}\left\|X(t)-X^{N}(t)\right\|_{L^{p}(\Omega ; H)} \leq C\left(1+\left\|X_{0}\right\|_{L^{12 p}(\Omega, V)}^{12}+\left\|X_{0}\right\|_{L^{4 p}\left(\Omega, \dot{H}^{\beta \vee[(p-1) /(2 p)]}\right)}^{4}+\left\|A^{\frac{\beta-1}{2}}\right\|_{\mathscr{L}_{2}}^{2}\right) N^{-\beta} .
$$

Here and below we denote $a \vee b:=\max (a, b)$. Clearly, the above convergence rate $\beta<\frac{1}{2}$ can be arbitrarily close to $\frac{1}{2}$ but can not reach $\frac{1}{2}$, since the quantity $\left\|A^{\frac{\beta-1}{2}}\right\|_{\mathscr{L}_{2}}$, as calculated in (2.4), explodes when $\beta$ tends to $\frac{1}{2}$. This comment also applies to the full approximation error estimates in section 4 . 
Proof of Theorem 3.4. The triangle inequality along with (2.15), (3.2) provides us that

$$
\begin{aligned}
\left\|X(t)-X^{N}(t)\right\|_{L^{p}(\Omega, H)} \leq & \left\|\left(I-P_{N}\right) X(t)\right\|_{L^{p}(\Omega, H)}+\left\|P_{N} X(t)-X^{N}(t)\right\|_{L^{p}(\Omega, H)} \\
\leq & N^{-\beta}\|X(t)\|_{L^{p}\left(\Omega ; \dot{H}^{\beta}\right)}+\left\|e_{t}^{N}\right\|_{L^{p}(\Omega, H)} \\
\leq & C_{2}\left(1+\left\|X_{0}\right\|_{L^{p}\left(\Omega, \dot{H}^{\beta}\right)}+\left\|X_{0}\right\|_{L^{3 p}(\Omega, V)}^{3}+\left\|A^{\frac{\beta-1}{2}}\right\|_{\mathscr{L}_{2}}\right) N^{-\beta} \\
& +\left\|e_{t}^{N}\right\|_{L^{p}(\Omega, H)},
\end{aligned}
$$

where $e_{t}^{N}:=P_{N} X(t)-X^{N}(t)=\int_{0}^{t} E_{N}(t-s)\left[F_{N}(X(s))-F_{N}\left(X^{N}(s)\right)\right] \mathrm{d} s$ satisfies

$$
\frac{\mathrm{d}}{\mathrm{d} t} e_{t}^{N}=-A_{N} e_{t}^{N}+F_{N}(X(t))-F_{N}\left(X^{N}(t)\right)=-A e_{t}^{N}+F_{N}(X(t))-F_{N}\left(X^{N}(t)\right) .
$$

Therefore, using (2.5) and the Young inequality gives

$$
\begin{aligned}
& \frac{\mathrm{d}}{\mathrm{d} t}\left\|e_{t}^{N}\right\|^{p}=p\left\|e_{t}^{N}\right\|^{p-2}\left\langle e_{t}^{N},-A e_{t}^{N}+F(X(t))-F\left(X^{N}(t)\right)\right\rangle \\
& \quad \leq p\left\|e_{t}^{N}\right\|^{p-2}\left\langle e_{t}^{N}, F\left(P_{N} X(t)\right)-F\left(X^{N}(t)\right)\right\rangle+p\left\|e_{t}^{N}\right\|^{p-2}\left\langle e_{t}^{N}, F(X(t))-F\left(P_{N} X(t)\right)\right\rangle \\
& \quad \leq L_{0} p\left\|e_{t}^{N}\right\|^{p}+p\left\|e_{t}^{N}\right\|^{p-1}\left\|F(X(t))-F\left(P_{N} X(t)\right)\right\| \\
& \quad \leq\left(L_{0} p+p-1\right)\left\|e_{t}^{N}\right\|^{p}+\left\|F(X(t))-F\left(P_{N} X(t)\right)\right\|^{p} \\
& \quad \leq\left(L_{0} p+p-1\right)\left\|e_{t}^{N}\right\|^{p}+C\left(1+\|X(t)\|_{V}^{2 p}+\left\|P_{N} X(t)\right\|_{V}^{2 p}\right)\left\|\left(I-P_{N}\right) X(t)\right\|^{p} .
\end{aligned}
$$

Choosing $\gamma=\frac{p-1}{2 p} \in\left(\frac{p-2}{2 p}, \frac{1}{2}\right)$ in Lemma 3.3 and also considering (2.14), (2.15) and (3.2) assure

$$
\begin{aligned}
\mathbb{E}\left[\left\|e_{t}^{N}\right\|^{p}\right] \leq & C \int_{0}^{t} \mathbb{E}\left[\left\|e_{s}^{N}\right\|^{p}\right]+\mathbb{E}\left[\left(1+\|X(s)\|_{V}^{2 p}+\left\|P_{N} X(s)\right\|_{V}^{2 p}\right)\left\|\left(I-P_{N}\right) X(s)\right\|^{p}\right] \mathrm{d} s \\
\leq & C \int_{0}^{t} \mathbb{E}\left[\left\|e_{s}^{N}\right\|^{p}\right]+\left(1+\|X(s)\|_{L^{4 p}(\Omega, V)}^{2 p}+\left\|P_{N} X(s)\right\|_{L^{4 p}(\Omega, V)}^{2 p}\right)\left\|\left(I-P_{N}\right) X(s)\right\|_{L^{2 p}(\Omega, H)}^{p} \mathrm{~d} s \\
\leq & C \int_{0}^{t} \mathbb{E}\left[\left\|e_{s}^{N}\right\|^{p}\right] \mathrm{d} s+C N^{-p \beta} \sup _{s \in[0, T]}\|X(s)\|_{L^{2 p}\left(\Omega, \dot{H}^{\beta}\right)}^{p} \\
& \times \int_{0}^{t}\left(1+\left\|X_{0}\right\|_{L^{12 p}(\Omega, V)}^{6 p}+s^{-\frac{1}{2}}\left\|X_{0}\right\|_{L^{4 p}\left(\Omega, \dot{H}^{(p-1) /(2 p)}\right)}^{2 p}\right) \mathrm{d} s \\
\leq & C \int_{0}^{t} \mathbb{E}\left[\left\|e_{s}^{N}\right\|^{p}\right] \mathrm{d} s+C N^{-p \beta}\left(1+\left\|X_{0}\right\|_{L^{p}\left(\Omega, \dot{H}^{\beta}\right)}+\left\|X_{0}\right\|_{L^{3 p}(\Omega, V)}^{3}+\left\|A^{\frac{\beta-1}{2}}\right\| \mathscr{L}_{2}\right)^{p} \\
& \times\left(1+\left\|X_{0}\right\|_{L^{12 p}(\Omega, V)}^{6 p}+\left\|X_{0}\right\|_{L^{4 p}\left(\Omega, \dot{H}^{(p-1) /(2 p))}\right.}^{2 p}\right) \\
\leq & C \int_{0}^{t} \mathbb{E}\left[\left\|e_{s}^{N}\right\|^{p}\right] \mathrm{d} s+C N^{-p \beta}\left(1+\left\|X_{0}\right\|_{L^{12 p}(\Omega, V)}^{6}+\left\|X_{0}\right\|_{L^{4 p}(\Omega, \dot{H} \beta}^{2 \beta V[(p-1) /(2 p)])}+\left\|A^{\frac{\beta-1}{2}}\right\|_{\mathscr{L}_{2}}\right)^{2 p} .
\end{aligned}
$$

After the use of the Gronwall inequality, one can derive from (3.14) the desired error bound.

\section{Spatio-temporal full discretization}

This section is devoted to error analysis of a spatio-temporal full discretization, done by a time discretization of the spatially discretized problem (3.3). For $M \in \mathbb{N}$ we construct a uniform mesh on $[0, T]$ with $\tau=\frac{T}{M}$ being the time stepsize, and propose a spatio-temporal full discretization as,

$$
Y_{t_{m+1}}^{M, N}=E_{N}(\tau) Y_{t_{m}}^{M, N}+\frac{A_{N}^{-1}\left(I-E_{N}(\tau)\right) F_{N}\left(Y_{t_{m}}^{M, N}\right)}{1+\tau\left\|F_{N}\left(Y_{t_{m}}^{M, N}\right)\right\|}+\int_{t_{m}}^{t_{m+1}} E_{N}\left(t_{m+1}-s\right) P_{N} \mathrm{~d} W(s)
$$


for $m=0,1, \ldots, M-1$ and $Y_{0}^{M, N}=P_{N} X_{0}$. Equivalently, the full discretization (4.1) can be written by $Y_{0}^{M, N}=P_{N} X_{0}$ and for $m=0,1, \ldots, M-1$,

$$
Y_{t_{m+1}}^{M, N}=E_{N}(\tau) Y_{t_{m}}^{M, N}+\int_{t_{m}}^{t_{m+1}} \frac{E_{N}\left(t_{m+1}-s\right) F_{N}\left(Y_{t_{m}}^{M, N}\right)}{1+\tau\left\|F_{N}\left(Y_{t_{m}}^{M, N}\right)\right\|} \mathrm{d} s+\int_{t_{m}}^{t_{m+1}} E_{N}\left(t_{m+1}-s\right) P_{N} \mathrm{~d} W(s) .
$$

Here we invoke a taming technique in $[25,48,47,23]$ for ordinary SDEs, and construct a nonlinearitytamed accelerated exponential Euler (AEE) scheme as (4.1). The so-called AEE scheme without taming is originally introduced in [30], to strongly approximate nearly linear parabolic SPDEs. Since the stochastic convolution is Gaussian distributed and diagonalizable on $\left\{e_{i}\right\}_{i \in \mathbb{N}}$, the scheme is much easier to simulate than it appears at first sight (see comments in section 5 for the implementation). When the nonlinearity grows super-linearly, one can in general not expect that the usual AEE schemes $[29,50,49,43,39,30]$ converge strongly, based on the observations from [24, 2]. Also, we mention that analyzing the strong convergence rate is much more difficult than that in the finite dimensional SDE setting.

When analyzing strong convergence rates, a crucial element is to derive uniform moment bounds for the spatio-temporal full discretization. To do this, a key ingredient lies on transforming the continuous time extension (4.19) of the full discretization (4.2) into a random differential equation (4.27), with a random perturbation appearing in the cubic nonlinear term. So the forthcoming subsection attempts to reveal that the $V$-norm of the solution to the perturbed differential equation can be controlled by a norm of the perturbation (see Lemma 4.2). Based on this finding, one can further use the structure of the taming scheme and a certain bootstrap argument (Lemma 4.4 and (4.49)) to provide a priori moment bound of the approximation, as shown by Theorem 4.6 in subsection 4.2. Reecovering the higher temporal convergence rate of order almost $\frac{1}{2}$ also heavily hinges on commutativity properties of the nonlinearity (Lemma 4.9) and the improved temporal Hölder regularity of the solution in negative Sobolev space (Lemma 4.10), as presented in subsection 4.3. Armed with these useful results, the expected convergence rate is finally proved in subsection 4.4 .

\subsection{Useful estimates for a perturbed differential equation}

In the first part, we restrict ourselves to the following perturbed differential equation in $H^{N}, N \in \mathbb{N}$,

$$
\left\{\begin{array}{l}
\frac{\partial v^{N}}{\partial t}=-A_{N} v^{N}+P_{N} F\left(v^{N}+z^{N}\right), \quad t \in(0, T] \\
v^{N}(0)=0
\end{array}\right.
$$

where $F$ comes from Assumption 2.2 and $z^{N}, v^{N}:[0, T] \rightarrow H^{N}$. In what follows, we aim to show that the $V$-norm of the solution to the perturbed problem (4.3) can be controlled by the $\mathbb{L}^{9}$-norm of the perturbation $z^{N}$ in the nonlinear mapping (see Lemma 4.2), which plays a crucial role in deducing uniform moment bounds for the full discretization (4.1). To show this, we need further smoothing properties of the analytic semigroup $E(t), t>0$ described as follows.

Lemma 4.1. Let $t>0$ and let $P_{N}, E(t)$ be defined as in the above sections. Then it holds that

$$
\begin{gathered}
\left\|P_{N} E(t) \psi\right\|_{L^{p}(D)} \leq t^{-\frac{p-2}{4 p}}\|\psi\|, \quad \forall p \geq 2, \\
\left\|P_{N} E(t) \psi\right\|_{V} \leq\left(\frac{t}{2}\right)^{-\frac{1}{2}}\|\psi\|_{L^{1}(D)}, \\
\left\|P_{N} E(t) \psi\right\|_{L^{3}(D)} \leq\left(\frac{t}{2}\right)^{-\frac{5}{24}}\|\psi\|_{L^{\frac{4}{3}}(D)} .
\end{gathered}
$$

Proof of Lemma 4.1. Recalling (3.9) with $\gamma=0$ helps us to infer,

$$
\left\|P_{N} E(t) \psi\right\|_{L^{p}(D)}^{p} \leq\left\|P_{N} E(t) \psi\right\|^{2} \cdot\left\|P_{N} E(t) \psi\right\|_{V}^{p-2} \leq\|\psi\|^{2} \cdot t^{-\frac{p-2}{4}}\|\psi\|^{p-2}=t^{-\frac{p-2}{4}}\|\psi\|^{p},
$$


which validates the first assertion. To arrive at the second one, we again use (3.9) with $\gamma=0$ to get

$$
\begin{aligned}
\left\|P_{N} E(t) \psi\right\|_{V} & =\left\|P_{N} E\left(\frac{t}{2}\right) P_{N} E\left(\frac{t}{2}\right) \psi\right\|_{V} \leq\left(\frac{t}{2}\right)^{-\frac{1}{4}}\left\|P_{N} E\left(\frac{t}{2}\right) \psi\right\|=\left(\frac{t}{2}\right)^{-\frac{1}{4}} \sup _{\|\phi\| \leq 1}\left|\left\langle P_{N} E\left(\frac{t}{2}\right) \psi, \phi\right\rangle\right| \\
& =\left(\frac{t}{2}\right)^{-\frac{1}{4}} \sup _{\|\phi\| \leq 1}\left|\left\langle\psi, P_{N} E\left(\frac{t}{2}\right) \phi\right\rangle\right| \leq\left(\frac{t}{2}\right)^{-\frac{1}{4}} \sup _{\|\phi\| \leq 1}\|\psi\|_{L^{1}(D)} \cdot\left\|P_{N} E\left(\frac{t}{2}\right) \phi\right\|_{V} \\
& \leq\left(\frac{t}{2}\right)^{-\frac{1}{2}}\|\psi\|_{L^{1}(D)} .
\end{aligned}
$$

Concerning the last inequality, one can similarly acquire

$$
\begin{aligned}
\left\|P_{N} E(t) \psi\right\|_{L^{3}(D)} & \leq\left(\frac{t}{2}\right)^{-\frac{1}{12}}\left\|P_{N} E\left(\frac{t}{2}\right) \psi\right\|=\left(\frac{t}{2}\right)^{-\frac{1}{12}} \sup _{\|\phi\| \leq 1}\left|\left\langle P_{N} E\left(\frac{t}{2}\right) \psi, \phi\right\rangle\right| \\
& =\left(\frac{t}{2}\right)^{-\frac{1}{12}} \sup _{\|\phi\| \leq 1}\left|\left\langle\psi, P_{N} E\left(\frac{t}{2}\right) \phi\right\rangle\right| \leq\left(\frac{t}{2}\right)^{-\frac{1}{12}} \sup _{\|\phi\| \leq 1}\|\psi\|_{L^{\frac{4}{3}}(D)}\left\|P_{N} E\left(\frac{t}{2}\right) \phi\right\|_{L^{4}(D)} \\
& \leq\left(\frac{t}{2}\right)^{-\frac{5}{24}}\|\psi\|_{L^{\frac{4}{3}}(D)} .
\end{aligned}
$$

The proof is now completed.

It is easy to see, the perturbed problem (4.3) has a unique solution in $H^{N}$, which can be expressed by

$$
v^{N}(t)=\int_{0}^{t} E_{N}(t-s) P_{N} F\left(v^{N}(s)+z^{N}(s)\right) \mathrm{d} s .
$$

Define norms $\|u\|_{\mathbb{L}^{q}(D \times[0, t])}:=\left(\int_{0}^{t}\|u(s)\|_{L^{q}(D)}^{q} \mathrm{~d} s\right)^{\frac{1}{q}}, q \geq 1, t \in[0, T]$. For the particular case $q=2$, $\mathbb{L}^{q}(D \times[0, t])\left(\mathbb{L}^{q}\right.$ for brevity) becomes a Hilbert space with $\langle u, v\rangle_{\mathbb{L}^{2}(D \times[0, t])}:=\int_{0}^{t}\langle u(s), v(s)\rangle \mathrm{d} s$. The next lemma asserts that the $V$-norm of the solution to (4.3) can be controlled by the $\mathbb{L}^{9}$-norm of the perturbation $z^{N}$, which will be essentially used in proving moment bounds of the approximations.

Lemma 4.2. Let $v^{N}, N \in \mathbb{N}$ be the solution to (4.3). For any $t \in[0, T]$, there exists a positive constant $C$, dependent of $T$ but independent of $N$, such that

$$
\left\|v^{N}(t)\right\|_{V} \leq C\left(1+\left\|z^{N}\right\|_{\mathbb{L}^{9}(D \times[0, t])}^{9}\right), \quad \forall t \in[0, T] .
$$

Proof of Lemma 4.2. The assertion is trivial for $t=0$. So we always suppose $t>0$ in the following. We divide the proof into two steps.

Step 1. For any fixed $t \in(0, T]$, we claim first that, by setting $\rho_{t}:=5 t^{\frac{1}{4}} \max \left\{\left.\frac{\left|a_{2}\right|}{a_{3}}|,| \frac{a_{1}}{a_{3}}\right|^{\frac{1}{2}},\left|\frac{a_{0}}{a_{3}}\right|^{\frac{1}{3}}\right\}$,

$$
\left\|v^{N}\right\|_{\mathbb{L}^{4}(D \times[0, t])} \leq 5\left\|z^{N}\right\|_{\mathbb{L}^{4}(D \times[0, t])} \quad \text { or } \quad\left\|v^{N}\right\|_{\mathbb{L}^{4}(D \times[0, t])} \leq \rho_{t} .
$$

By deterministic calculus and noting $A_{N} v^{N}=A v^{N}$ for any $v^{N} \in H^{N}$, we derive

$$
\begin{aligned}
0 & \leq \frac{1}{2}\left\|v^{N}(t)\right\|^{2}=\int_{0}^{t}\left\langle v^{N}(s),-A_{N} v^{N}(s)+P_{N} F\left(v^{N}(s)+z^{N}(s)\right)\right\rangle \mathrm{d} s \\
& \leq \int_{0}^{t}\left\langle v^{N}(s), F\left(v^{N}(s)+z^{N}(s)\right)\right\rangle \mathrm{d} s=\left\langle v^{N}, F\left(v^{N}+z^{N}\right)\right\rangle_{\mathbb{L}^{2}} .
\end{aligned}
$$

Noticing that $a_{3}<0$, for any $v, z \in \mathbb{R}$,

$$
\begin{aligned}
v f(v+z) & =a_{3} v(v+z)^{3}+a_{2} v(v+z)^{2}+a_{1} v(v+z)+a_{0} v \\
& \leq a_{3} v^{4}+3 a_{3} v^{3} z+a_{3} v z^{3}+a_{2} v^{3}+2 a_{2} v^{2} z+a_{2} v z^{2}+a_{1} v^{2}+a_{1} v z+a_{0} v .
\end{aligned}
$$

After using the fact $a_{3}<0$ and the Hölder inequality, one derives

$$
\begin{aligned}
\left\langle v^{N}, F\left(v^{N}+z^{N}\right)\right\rangle_{\mathbb{L}^{2}} \leq & a_{3}\left\|v^{N}\right\|_{\mathbb{L}^{4}}^{4}+3\left|a_{3}\right|\left\|v^{N}\right\|_{\mathbb{L}^{4}}^{3}\left\|z^{N}\right\|_{\mathbb{L}^{4}}+\left|a_{3}\right|\left\|v^{N}\right\|_{\mathbb{L}^{4}}\left\|z^{N}\right\|_{\mathbb{L}^{4}}^{3} \\
& +\left|a_{2}\right| t^{\frac{1}{4}}\left\|v^{N}\right\|_{\mathbb{L}^{4}}^{3}+2\left|a_{2}\right| t^{\frac{1}{4}}\left\|v^{N}\right\|_{\mathbb{L}^{4}}^{2}\left\|z^{N}\right\|_{\mathbb{L}^{4}}+\left|a_{2}\right| t^{\frac{1}{4}}\left\|v^{N}\right\|_{\mathbb{L}^{4}}\left\|z^{N}\right\|_{\mathbb{L}^{4}}^{2} \\
& +\left|a_{1}\right| t^{\frac{1}{2}}\left\|v^{N}\right\|_{\mathbb{L}^{4}}^{2}+\left|a_{1}\right| t^{\frac{1}{2}}\left\|v^{N}\right\|_{\mathbb{L}^{4}}\left\|z^{N}\right\|_{\mathbb{L}^{4}}+\left|a_{0}\right| t^{\frac{3}{4}}\left\|v^{N}\right\|_{\mathbb{L}^{4}} .
\end{aligned}
$$


Assume the claim (4.10) is false, namely, $\left\|z^{N}\right\|_{\mathbb{L}^{4}(D \times[0, t])}<\frac{1}{5}\left\|v^{N}\right\|_{\mathbb{L}^{4}(D \times[0, t])}$ and $\left\|v^{N}\right\|_{\mathbb{L}^{4}(D \times[0, t])}>\rho_{t}$. This enables us to derive, in the case $\rho_{t}>0$, i.e., $\left|a_{0}\right|+\left|a_{1}\right|+\left|a_{2}\right|>0$,

$$
\begin{aligned}
\left\langle v^{N}, F\left(v^{N}+z^{N}\right)\right\rangle_{\mathbb{L}^{2}}< & \left(a_{3}+\frac{3\left|a_{3}\right|}{5}+\frac{\left|a_{3}\right|}{125}\right)\left\|v^{N}\right\|_{\mathbb{L}^{4}}^{4}+\left(\left|a_{2}\right| t^{\frac{1}{4}}+\frac{2\left|a_{2}\right| t^{\frac{1}{4}}}{5}+\frac{\left|a_{2}\right| t^{\frac{1}{4}}}{25}\right)\left\|v^{N}\right\|_{\mathbb{L}^{4}}^{3} \\
& +\left(\left|a_{1}\right| t^{\frac{1}{2}}+\frac{\left|a_{1}\right| t^{\frac{1}{2}}}{5}\right)\left\|v^{N}\right\|_{\mathbb{L}^{4}}^{2}+\left|a_{0}\right| t^{\frac{3}{4}}\left\|v^{N}\right\|_{\mathbb{L}^{4}} \\
< & \left(a_{3}+\frac{76\left|a_{3}\right|}{125}+\frac{\left|a_{2}\right| t^{\frac{1}{4}}}{\rho_{t}}+\frac{2\left|a_{2}\right| t^{\frac{1}{4}}}{5 \rho_{t}}+\frac{\left|a_{2}\right| t^{\frac{1}{4}}}{25 \rho_{t}}+\frac{\left|a_{1}\right| t^{\frac{1}{2}}}{\rho_{t}^{2}}+\frac{\left|a_{1}\right| t^{\frac{1}{2}}}{5 \rho_{t}^{2}}+\frac{\left|a_{0}\right| t^{\frac{3}{4}}}{\rho_{t}^{3}}\right)\left\|v^{N}\right\|_{\mathbb{L}^{4}}^{4} \\
\leq & \left(a_{3}+\frac{76\left|a_{3}\right|}{125}+\frac{\left|a_{3}\right|}{5}+\frac{2\left|a_{3}\right|}{25}+\frac{\left|a_{3}\right|}{125}+\frac{\left|a_{3}\right|}{25}+\frac{\left|a_{3}\right|}{125}+\frac{\left|a_{3}\right|}{125}\right)\left\|v^{N}\right\|_{\mathbb{L}^{4}}^{4} \\
= & \frac{6 a_{3}}{125}\left\|v^{N}\right\|_{\mathbb{L}^{4}}^{4}<0,
\end{aligned}
$$

where we set $\rho_{t}:=5 t^{\frac{1}{4}} \max \left\{\frac{\left|a_{2}\right|}{\left|a_{3}\right|},\left|\frac{a_{1}}{a_{3}}\right|^{\frac{1}{2}},\left|\frac{a_{0}}{a_{3}}\right|^{\frac{1}{3}}\right\}$ such that all coefficients of $\left\|v^{N}\right\|_{\mathbb{L}^{4}}^{4}$ in the second step only consist of $\left|a_{3}\right|$ or $a_{3}$ and the resulting collection turns out to be negative. The conclusion (4.14) thus contradicts (4.11). When $a_{0}=a_{1}=a_{2}=0$, i.e., $\rho_{t}=0$, one can similarly derive $\left\langle v^{N}, F\left(v^{N}+z^{N}\right)\right\rangle_{\mathbb{L}^{2}}<$ $\left(a_{3}+\frac{3\left|a_{3}\right|}{5}+\frac{\left|a_{3}\right|}{125}\right)\left\|v^{N}\right\|_{\mathbb{L}^{4}}^{4}=\frac{49 a_{3}}{125}\left\|v^{N}\right\|_{\mathbb{L}^{4}}^{4} \leq 0$, also contradicting (4.11). Therefore, the claim (4.10) must be true.

Step 2. Apparently, (4.10) implies

$$
\left\|v^{N}\right\|_{\mathbb{L}^{4}(D \times[0, t])} \leq 5\left\|z^{N}\right\|_{\mathbb{L}^{4}(D \times[0, t])}+\rho_{T}, \quad \forall t \in[0, T] .
$$

This together with the last inequality in (4.4), the property of the cubic nonlinearity and the Hölder inequality yields, for any $t \in[0, T]$,

$$
\begin{aligned}
\left\|v^{N}(t)\right\|_{L^{3}(D)} & \leq \int_{0}^{t}\left\|E(t-s) P_{N} F\left(v^{N}(s)+z^{N}(s)\right)\right\|_{L^{3}(D)} \mathrm{d} s \\
& \leq \int_{0}^{t}\left(\frac{t-s}{2}\right)^{-\frac{5}{24}}\left\|F\left(v^{N}(s)+z^{N}(s)\right)\right\|_{L^{\frac{4}{3}}(D)} \mathrm{d} s \\
& \leq C \int_{0}^{t}\left(\frac{t-s}{2}\right)^{-\frac{5}{24}}\left(1+\left\|v^{N}(s)\right\|_{L^{4}(D)}^{3}+\left\|z^{N}(s)\right\|_{L^{4}(D)}^{3}\right) \mathrm{d} s \\
& \leq C\left(\int_{0}^{t}\left(\frac{t-s}{2}\right)^{-\frac{5}{6}} \mathrm{~d} s\right)^{\frac{1}{4}}\left(\int_{0}^{t} 1+\left\|v^{N}(s)\right\|_{L^{4}(D)}^{4}+\left\|z^{N}(s)\right\|_{L^{4}(D)}^{4} \mathrm{~d} s\right)^{\frac{3}{4}} \\
& \leq C\left(1+\left\|z^{N}\right\|_{\mathbb{L}^{4}(D \times[0, t])}^{3}\right) .
\end{aligned}
$$

Likewise, by virtue of the second inequality in (4.4) instead, one obtains, for any $t \in[0, T]$,

$$
\begin{aligned}
\left\|v^{N}(t)\right\|_{V} & \leq \int_{0}^{t}\left\|P_{N} E(t-s) F\left(v^{N}(s)+z^{N}(s)\right)\right\|_{V} \mathrm{~d} s \\
& \leq C \int_{0}^{t}\left(\frac{t-s}{2}\right)^{-\frac{1}{2}}\left\|F\left(v^{N}(s)+z^{N}(s)\right)\right\|_{L^{1}(D)} \mathrm{d} s \\
& \leq C \int_{0}^{t}(t-s)^{-\frac{1}{2}}\left(1+\left\|v^{N}(s)\right\|_{L^{3}(D)}^{3}+\left\|z^{N}(s)\right\|_{L^{3}(D)}^{3}\right) \mathrm{d} s \\
& \leq C\left(1+\left\|z^{N}\right\|_{\mathbb{L}^{4}(D \times[0, t])}^{9}+\int_{0}^{t}(t-s)^{-\frac{1}{2}}\left\|z^{N}(s)\right\|_{L^{3}(D)}^{3} \mathrm{~d} s\right) \\
& \leq C\left(1+\left\|z^{N}\right\|_{\mathbb{L}^{9}(D \times[0, t])}^{9}\right) .
\end{aligned}
$$

The proof of Lemma 4.2 is thus finished.

Before closing this subsection, we would like to add some comments on the proof of Lemma 4.2. The key step is to show either $\left\|v^{N}\right\|_{\mathbb{L}^{4}(D \times[0, t])} \leq \kappa\left\|z^{N}\right\|_{\mathbb{L}^{4}(D \times[0, t])}$ or $\left\|v^{N}\right\|_{\mathbb{L}^{4}(D \times[0, t])} \leq \rho_{t}$, with some positive constants $\kappa, \rho_{t}$ properly chosen. As shown above, its proof is based on the reduction to absurdity and we choose $\kappa=5$ and $\rho_{t}:=5 t^{\frac{1}{4}} \max \left\{\frac{\left|a_{2}\right|}{a_{3}},\left|\frac{a_{1}}{a_{3}}\right|^{\frac{1}{2}},\left|\frac{a_{0}}{a_{3}}\right|^{\frac{1}{3}}\right\}$ in (4.14) to result in a contradiction. We mention that the choice of $\kappa, \rho_{t}$ is, however, not unique. 


\subsection{A priori moment bounds of the approximations}

The aim of this subsection is to prove a priori moment bounds for the fully discrete approximation (4.1) (or (4.2) equivalently), which is essentially based on the estimate (4.9) obtained in the previous subsection as well as a certain bootstrap argument. First, we define

$$
\lfloor t\rfloor:=t_{i}=i \tau, \quad \text { for } t \in\left[t_{i}, t_{i+1}\right), \quad i \in\{0,1, \ldots, M-1\},
$$

and introduce a continuous version of the fully discrete scheme (4.2) as,

$$
Y_{t}^{M, N}=E_{N}(t) Y_{0}^{M, N}+\int_{0}^{t} \frac{E_{N}(t-s) F_{N}\left(Y_{[s}^{M, N}\right)}{1+\tau\left\|F_{N}\left(Y_{\lfloor s]}^{M, N}\right)\right\|} \mathrm{d} s+\mathscr{O}_{t}^{N}, \quad t \in[0, T]
$$

Here we recall that $\tau=\frac{T}{M}$ is the uniform time stepsize. By $B^{c}$ and $\mathbb{1}_{B}$, we denote the complement and indicator function of a set $B$, respectively. Additionally, we introduce a sequence of decreasing subevents

$$
\Omega_{R, t_{i}}:=\left\{\omega \in \Omega: \sup _{j \in\{0,1, \ldots, i\}}\left\|Y_{t_{j}}^{M, N}(\omega)\right\|_{V} \leq R\right\}, \quad R \in(0, \infty), i \in\{0,1, \ldots, M\} .
$$

It is clear that $\mathbb{1}_{\Omega_{R, t_{i}}} \in \mathscr{F}_{t_{i}}$ for $i \in\{0,1, \ldots, M\}$ and $\mathbb{1}_{\Omega_{R, t_{i}}} \leq \mathbb{1}_{\Omega_{R, t_{j}}}$ for $t_{i} \geq t_{j}$ since $\Omega_{R, t_{i}} \subset \Omega_{R, t_{j}}, t_{i} \geq t_{j}$. Furthermore, we put additional assumptions on the initial data.

Assumption 4.3. For sufficiently large positive number $p_{0} \in \mathbb{N}$, the initial data $X_{0}$ obeys

$$
\sup _{N \in \mathbb{N}}\left\|P_{N} X_{0}\right\|_{L^{p_{0}(\Omega, V)}}<\infty .
$$

Thanks to the Sobolev embedding inequality, (4.21) is fulfilled provided $\left\|P_{N} X_{0}\right\|_{L^{p_{0}\left(\Omega, \dot{H}^{\gamma}\right)}}<\infty$ for any $\gamma>\frac{1}{2}$. In what follows we start the bootstrap argument, by showing $\mathbb{E}\left[\mathbb{1}_{\Omega_{R^{\tau}, t_{m}}}\left\|Y_{t_{m}}^{M, N}\right\|_{V}^{p}\right]<\infty$ and $\mathbb{E}\left[\mathbb{1}_{\Omega_{R^{\tau}, t_{m}}^{c}}\left\|Y_{t_{m}}^{M, N}\right\|_{V}^{p}\right]<\infty$ for subevents $\Omega_{R^{\tau}, t}$ with $R^{\tau}$ depending on $\tau$ carefully chosen.

Lemma 4.4. Let $p \in[2, \infty)$ and $R^{\tau}:=\tau^{-\frac{\beta}{4}}$ for any $\beta \in\left(0, \frac{1}{2}\right)$. Under Assumptions $2.1-2.4,4.3$, the approximation process $Y_{t_{i}}^{M, N}, i \in\{0,1, \ldots, M\}$ produced by (4.1) obeys

$$
\sup _{M, N \in \mathbb{N}} \sup _{i \in\{0,1, \ldots, M\}} \mathbb{E}\left[\mathbb{1}_{\Omega_{R^{\tau}, t_{i-1}}}\left\|Y_{t_{i}}^{M, N}\right\|_{V}^{p}\right]<\infty,
$$

where we set $\mathbb{1}_{\Omega_{R^{\tau}, t_{-1}}}=1$.

Proof of Lemma 4.4. The proof heavily relies on the use of Lemma 4.2. In order to apply it, we introduce a process $Z_{t}^{M, N}$ given by,

$$
\begin{aligned}
Z_{t}^{M, N}:= & E_{N}(t) Y_{0}^{M, N}+\int_{0}^{t} E_{N}(t-s)\left[\frac{P_{N} F\left(Y_{\mid s\rfloor}^{M, N}\right)}{1+\tau\left\|P_{N} F\left(Y_{[s\rfloor}^{M, N}\right)\right\|}-P_{N} F\left(Y_{s}^{M, N}\right)\right] \mathrm{d} s+\mathscr{O}_{t}^{N} \\
= & E_{N}(t) Y_{0}^{M, N}+\int_{0}^{t} E(t-s) P_{N}\left[F\left(Y_{\lfloor s\rfloor}^{M, N}\right)-F\left(Y_{s}^{M, N}\right)\right] \mathrm{d} s \\
& +\int_{0}^{t} E(t-s)\left[\frac{P_{N} F\left(Y_{[s\rfloor}^{M, N}\right)}{1+\tau\left\|P_{N} F\left(Y_{\lfloor s\rfloor}^{M, N}\right)\right\|}-P_{N} F\left(Y_{\lfloor s\rfloor}^{M, N}\right)\right] \mathrm{d} s+P_{N} \mathscr{O}_{t}, \quad t \in[0, T] .
\end{aligned}
$$

Bearing this in mind, one can rewrite (4.19) as

$$
Y_{t}^{M, N}=\int_{0}^{t} E_{N}(t-s) P_{N} F\left(Y_{s}^{M, N}\right) \mathrm{d} s+Z_{t}^{M, N} .
$$


Further, we define $\bar{Y}_{t}^{M, N}$ as

$$
\bar{Y}_{t}^{M, N}:=Y_{t}^{M, N}-Z_{t}^{M, N}, \quad \text { with } \quad \bar{Y}_{0}^{M, N}=0 .
$$

So, we recast (4.24) as

$$
\bar{Y}_{t}^{M, N}=\int_{0}^{t} E_{N}(t-s) P_{N} F\left(\bar{Y}_{s}^{M, N}+Z_{s}^{M, N}\right) \mathrm{d} s, \quad t \in[0, T],
$$

which satisfies

$$
\frac{\mathrm{d}}{\mathrm{d} t} \bar{Y}_{t}^{M, N}=-A_{N} \bar{Y}_{t}^{M, N}+P_{N} F\left(\bar{Y}_{t}^{M, N}+Z_{t}^{M, N}\right), \quad t \in(0, T], \quad \bar{Y}_{0}^{M, N}=0 .
$$

Now one can employ Lemma 4.2 to obtain,

$$
\left\|\bar{Y}_{t}^{M, N}\right\|_{V} \leq C\left(1+\left\|Z^{M, N}\right\|_{\mathbb{L}^{9}(D \times[0, t])}^{9}\right), \quad t \in[0, T],
$$

where $Z^{M, N}$ is defined by (4.23). Thus, for any $i \in\{0,1, \ldots, M\}$,

$$
\begin{aligned}
\mathbb{E}\left[\mathbb{1}_{\Omega_{R, t_{i-1}}}\left\|\bar{Y}_{t_{i}}^{M, N}\right\|_{V}^{p}\right] & \leq C\left(1+\mathbb{E}\left[\mathbb{1}_{\Omega_{R, t_{i-1}}}\left\|Z^{M, N}\right\|_{\mathbb{L}^{9 p}\left(D \times\left[0, t_{i}\right]\right.}^{9 p}\right]\right) \\
& \leq C\left(1+\mathbb{E}\left[\mathbb{1}_{\Omega_{R, t_{i-1}}} \int_{0}^{t_{i}}\left\|Z_{s}^{M, N}\right\|_{V}^{9 p} \mathrm{~d} s\right]\right),
\end{aligned}
$$

where, for $s \in\left[0, t_{i}\right], i \in\{0,1, \ldots, M\}$, it holds that

$$
\begin{aligned}
\mathbb{1}_{\Omega_{R, t_{i-1}}\left\|Z_{s}^{M, N}\right\|_{V} \leq} & \left\|E_{N}(s) Y_{0}^{M, N}\right\|_{V}+\mathbb{1}_{\Omega_{R, t_{i-1}}}\left\|\int_{0}^{s} E(s-r) P_{N}\left[F\left(Y_{r}^{M, N}\right)-F\left(Y_{\lfloor r\rfloor}^{M, N}\right)\right] \mathrm{d} r\right\|_{V} \\
& +\mathbb{1}_{\Omega_{R, t_{i-1}}}\left\|\int_{0}^{s} E(s-r) P_{N} F\left(Y_{\lfloor r\rfloor}^{M, N}\right) \frac{\tau\left\|P_{N} F\left(Y_{\lfloor r !}^{M, N}\right)\right\|}{1+\tau\left\|P_{N} F\left(Y_{\lfloor r\rfloor}^{M, N}\right)\right\|} \mathrm{d} r\right\|_{V}+\left\|P_{N} \mathscr{O}_{s}\right\|_{V} \\
= & :\left\|E_{N}(s) Y_{0}^{M, N}\right\|_{V}+I_{1}+I_{2}+\left\|P_{N} \mathscr{O}_{s}\right\|_{V} .
\end{aligned}
$$

Before proceeding further, we claim

$$
\mathbb{1}_{\Omega_{R, t_{i-1}}}\left\|Y_{r}^{M, N}\right\|_{V} \leq C\left(1+R+\tau^{\frac{3}{4}} R^{3}+\left\|\mathscr{O}_{r}^{N}\right\|_{V}+\left\|\mathscr{O}_{\lfloor r\rfloor}^{N}\right\|_{V}\right), \quad \forall r \in\left[0, t_{i}\right) .
$$

By the definition of $Y_{r}^{M, N}$ and noting $\int_{\lfloor r\rfloor}^{r} E(r-u) P_{N} \mathrm{~d} W_{u}=\mathscr{O}_{r}^{N}-E(r-\lfloor r\rfloor) \mathscr{O}_{\lfloor r\rfloor}^{N}, r \in\left[0, t_{i}\right)$, one can write

$$
Y_{r}^{M, N}=E(r-\lfloor r\rfloor) Y_{\lfloor r\rfloor}^{M, N}+\int_{\lfloor r\rfloor}^{r} \frac{E(r-u) F_{N}\left(Y_{\lfloor u\rfloor}^{M, N}\right)}{1+\tau\left\|F_{N}\left(Y_{\lfloor u\rfloor}^{M, N}\right)\right\|} \mathrm{d} u+\mathscr{O}_{r}^{N}-E(r-\lfloor r\rfloor) \mathscr{O}_{\lfloor r\rfloor}^{N} .
$$

This together with the boundedness of the semigroup $E(t)$ in $V$, i.e., $\|E(t) \phi\|_{V} \leq\|\phi\|_{V}$ and (3.9) for $\gamma=0$ promises

$$
\begin{aligned}
\mathbb{1}_{\Omega_{R, t_{i-1}}}\left\|Y_{r}^{M, N}\right\|_{V} \leq & \mathbb{1}_{\Omega_{R, t_{i-1}}}\left(\left\|E(r-\lfloor r\rfloor) Y_{\lfloor r\rfloor}^{M, N}\right\|_{V}+\int_{\lfloor r\rfloor}^{r}\left\|E(r-u) F_{N}\left(Y_{\lfloor u\rfloor}^{M, N}\right)\right\|_{V} \mathrm{~d} u\right. \\
& \left.+\left\|\mathscr{O}_{r}^{N}-E(r-\lfloor r\rfloor) \mathscr{O}_{\lfloor r\rfloor}^{N}\right\|_{V}\right) \\
\leq & \mathbb{1}_{\Omega_{R, t_{i-1}}}\left(\left\|Y_{\lfloor r\rfloor}^{M, N}\right\|_{V}+\int_{\lfloor r\rfloor}^{r}(r-u)^{-\frac{1}{4}}\left\|F\left(Y_{\lfloor u\rfloor}^{M, N}\right)\right\| \mathrm{d} u+\left\|\mathscr{O}_{r}^{N}\right\|_{V}+\left\|\mathscr{O}_{\lfloor r\rfloor}^{N}\right\|_{V}\right) \\
\leq & C\left(1+R+\frac{4}{3} \tau^{3 / 4} R^{3}+\left\|\mathscr{O}_{r}^{N}\right\|_{V}+\left\|\mathscr{O}_{\lfloor r\rfloor}^{N}\right\|_{V}\right) .
\end{aligned}
$$

This shows the claim (4.31). With the aid of (2.5) and (4.31), the first term $I_{1}$ can be treated as follows,

$$
\begin{aligned}
I_{1} & \leq \mathbb{1}_{\Omega_{R, t_{i-1}}} \int_{0}^{s}(s-r)^{-\frac{1}{4}}\left\|F\left(Y_{r}^{M, N}\right)-F\left(Y_{\lfloor r\rfloor}^{M, N}\right)\right\| \mathrm{d} r \\
& \leq \mathbb{1}_{\Omega_{R, t_{i-1}}} C \int_{0}^{s}(s-r)^{-\frac{1}{4}}\left(1+R^{2}+\tau^{\frac{3}{2}} R^{6}+\left\|\mathscr{O}_{r}^{N}\right\|_{V}^{2}+\left\|\mathscr{O}_{\lfloor r\rfloor}^{N}\right\|_{V}^{2}\right)\left\|Y_{r}^{M, N}-Y_{\lfloor r\rfloor}^{M, N}\right\| \mathrm{d} r,
\end{aligned}
$$


where $r \in[0, s], s \in\left[0, t_{i}\right]$,

$$
\begin{aligned}
Y_{r}^{M, N}-Y_{\lfloor r\rfloor}^{M, N}= & {[E(r)-E(\lfloor r\rfloor)] Y_{0}^{M, N}+\int_{0}^{r} E(r-u) \frac{P_{N} F\left(Y_{\lfloor\rfloor\rfloor}^{M, N}\right)}{1+\tau\left\|P_{N} F\left(Y_{\lfloor u\rfloor}^{M, N}\right)\right\|} \mathrm{d} u } \\
& -\int_{0}^{\lfloor r\rfloor} E(\lfloor r\rfloor-u) \frac{P_{N} F\left(Y_{\lfloor u\rfloor}^{M, N}\right)}{1+\tau\left\|P_{N} F\left(Y_{\lfloor u\rfloor}^{M, N}\right)\right\|} \mathrm{d} u+P_{N} \mathscr{O}_{r}-P_{N} \mathscr{O}_{\lfloor r\rfloor} .
\end{aligned}
$$

This ensures that

$$
\begin{aligned}
& \mathbb{1}_{\Omega_{R, t_{i-1}}}\left\|Y_{r}^{M, N}-Y_{\lfloor r\rfloor}^{M, N}\right\| \\
& \leq \tau^{\frac{\beta}{2}}\left\|Y_{0}^{M, N}\right\|_{\beta}+1_{\Omega_{R, t_{i-1}}}\left\|\int_{0}^{\lfloor r\rfloor} E(\lfloor r\rfloor-u)(E(r-\lfloor r\rfloor)-I) \frac{P_{N} F\left(Y_{\lfloor u\rfloor}^{M, N}\right)}{1+\tau\left\|P_{N} F\left(Y_{\lfloor u\rfloor}^{M, N}\right)\right\|} \mathrm{d} u\right\| \\
& \quad+\mathbb{1}_{\Omega_{R, t_{i-1}}}\left\|\int_{\lfloor r\rfloor}^{r} E(r-u) \frac{P_{N} F\left(Y_{\lfloor u\rfloor}^{M, N}\right)}{1+\tau\left\|P_{N} F\left(Y_{\lfloor u\rfloor}^{M, N}\right)\right\|} \mathrm{d} u\right\|+\mathbb{1}_{\Omega_{R, t_{i-1}}}\left\|P_{N}\left(\mathscr{O}_{r}-\mathscr{O}_{\lfloor r\rfloor}\right)\right\| \\
& \leq \tau^{\frac{\beta}{2}}\left\|X_{0}\right\|_{\beta}+C\left(1+R^{3}\right)\left(\tau^{\frac{3}{4}}+\tau\right)+\left\|\mathscr{O}_{r}-\mathscr{O}_{\lfloor r\rfloor}\right\| .
\end{aligned}
$$

Inserting this into (4.34) results in

$$
\begin{aligned}
I_{1} \leq & C \int_{0}^{s}\left(1+R^{2}+\tau^{\frac{3}{2}} R^{6}+\left\|\mathscr{O}_{r}^{N}\right\|_{V}^{2}+\left\|\mathscr{O}_{\lfloor r\rfloor}^{N}\right\|_{V}^{2}\right)(s-r)^{-\frac{1}{4}}\left[\tau^{\frac{\beta}{2}}\left\|X_{0}\right\|_{\beta}+C\left(1+R^{3}\right) \tau^{\frac{3}{4}}+\left\|\mathscr{O}_{r}-\mathscr{O}_{\lfloor r\rfloor}\right\|\right] \mathrm{d} r \\
= & C \tau^{\frac{\beta}{2}}\left\|X_{0}\right\|_{\beta} \int_{0}^{s}\left(1+R^{2}+\tau^{\frac{3}{2}} R^{6}+\left\|\mathscr{O}_{r}^{N}\right\|_{V}^{2}+\left\|\mathscr{O}_{\lfloor r\rfloor}^{N}\right\|_{V}^{2}\right)(s-r)^{-\frac{1}{4}} \mathrm{~d} r \\
& +C\left(1+R^{3}\right) \tau^{\frac{3}{4}} \int_{0}^{s}\left(1+R^{2}+\tau^{\frac{3}{2}} R^{6}+\left\|\mathscr{O}_{r}^{N}\right\|_{V}^{2}+\left\|\mathscr{O}_{\lfloor r\rfloor}^{N}\right\|_{V}^{2}\right)(s-r)^{-\frac{1}{4}} \mathrm{~d} r \\
& +C \int_{0}^{s}(s-r)^{-\frac{1}{4}}\left(1+R^{2}+\tau^{\frac{3}{2}} R^{6}+\left\|\mathscr{O}_{r}^{N}\right\|_{V}^{2}+\left\|\mathscr{O}_{\lfloor r\rfloor}^{N}\right\|_{V}^{2}\right)\left\|\mathscr{O}_{r}-\mathscr{O}_{\lfloor r\rfloor}\right\| \mathrm{d} r
\end{aligned}
$$

Therefore, letting $R=R^{\tau}:=\tau^{-\frac{\beta}{4}}$ and considering (2.11)-(2.12) one can further infer that

$$
\left\|I_{1}\right\|_{L^{9 p}(\Omega, \mathbb{R})} \leq C\left(1+\left\|X_{0}\right\|_{L^{9 p}\left(\Omega, \dot{H}^{\beta}\right)}+\left\|A^{\frac{\beta-1}{2}}\right\|_{\mathscr{L}_{2}(H)}\right) .
$$

In a similar manner, choosing $R=R^{\tau}:=\tau^{-\frac{\beta}{4}}$ enables us to treat $I_{2}$ as follows:

$$
\begin{aligned}
I_{2} & \leq \mathbb{1}_{\Omega_{R, t_{i-1}}} \int_{0}^{s}\left\|E(s-r) P_{N} F\left(Y_{\lfloor r\rfloor}^{M, N}\right)\right\|_{V} \cdot \tau\left\|P_{N} F\left(Y_{\lfloor r\rfloor}^{M, N}\right)\right\| \mathrm{d} r \\
& \leq \mathbb{1}_{\Omega_{R, t_{i-1}}} \tau \int_{0}^{s}(s-r)^{-\frac{1}{4}}\left\|F\left(Y_{\lfloor r\rfloor}^{M, N}\right)\right\|^{2} \mathrm{~d} r \\
& \leq C\left(R^{6}+1\right) \tau \\
& \leq C\left(\tau^{\frac{2-3 \beta}{2}}+\tau\right),
\end{aligned}
$$

where we also used (3.9) with $\gamma=0$ and the structure of the taming scheme, namely, $\frac{z}{1+\tau z} \leq z, z \geq 0$. Bearing (4.38), (4.39) and (3.5) in mind, one can deduce from (4.30) that, for any $s \in\left[0, t_{i}\right]$,

$$
\mathbb{E}\left[\mathbb{1}_{\Omega_{R, t_{i-1}}}\left\|Z_{s}^{M, N}\right\|_{V}^{9 p}\right] \leq C<\infty .
$$

This together with (4.29) and (4.40) immediately implies

$$
\mathbb{E}\left[\mathbb{1}_{\Omega_{R} \tau, t_{i-1}}\left\|\bar{Y}_{t_{i}}^{M, N}\right\|_{V}^{p}\right] \leq C<\infty .
$$

Combining this with (4.25) verifies the desired assertion (4.22).

As a direct consequence of Lemma 4.4, the following corollary offers a priori moment bounds of the numerical approximation on subevents $\Omega_{R^{\tau}, t_{i}}, i \in\{0,1, \ldots, M\}$. 
Corollary 4.5. Let $p \in[2, \infty)$ and $R^{\tau}:=\tau^{-\frac{\beta}{4}}$ for any $\beta \in\left(0, \frac{1}{2}\right)$. Let Assumptions 2.1-2.4, 4.3 hiold and let the approximation process $Y_{t_{i}}^{M, N}, i \in\{0,1, \ldots, M\}$ be produced by (4.1). Then it holds

$$
\sup _{M, N \in \mathbb{N}} \sup _{i \in\{0,1, \ldots, M\}} \mathbb{E}\left[\mathbb{1}_{\Omega_{R} \tau, t_{i}}\left\|Y_{t_{i}}^{M, N}\right\|_{V}^{p}\right]<\infty .
$$

Proof of Corollary 4.5. Thanks to the assertion (4.22) and the fact that $\Omega_{R, t_{i}} \subset \Omega_{R, t_{i-1}}$, one can easily deduce

$$
\sup _{M, N \in \mathbb{N}} \sup _{i \in\{0,1, \ldots, M\}} \mathbb{E}\left[\mathbb{1}_{\Omega_{R} \tau, t_{i}}\left\|Y_{t_{i}}^{M, N}\right\|_{V}^{p}\right] \leq \sup _{M, N \in \mathbb{N}} \sup _{i \in\{0,1, \ldots, M\}} \mathbb{E}\left[\mathbb{1}_{\Omega_{R^{\tau}, t_{i-1}}}\left\|Y_{t_{i}}^{M, N}\right\|_{V}^{p}\right]<\infty
$$

as required.

Equipped with the bounded moments (4.42) on subevents $\Omega_{R^{\tau}, t_{i}}, i \in\{0,1, \ldots, M\}$, one only needs to deduce bounded moments on its complement $\Omega_{R^{\tau}, t_{m}}^{c}$ before arriving at the expected bounded moments on the whole probability space stated as follows.

Theorem 4.6. Let Assumptions 2.1-2.4, 4.3 be fulfilled and let the approximation process $Y_{t_{i}}^{M, N}, i \in$ $\{0,1, \ldots, M\}$ be produced by (4.1). Then for any $p \in[2, \infty)$ it holds that

$$
\sup _{M, N \in \mathbb{N}} \sup _{m \in\{0,1, \ldots, M\}} \mathbb{E}\left[\left\|Y_{t_{m}}^{M, N}\right\|_{V}^{p}\right]<\infty .
$$

Proof of Theorem 4.6. As discussed above, it remains to bound $\sup _{M, N \in \mathbb{N}} \sup _{m \in\{0,1, \ldots, M\}} \mathbb{E}\left[\mathbb{1}_{\Omega_{R^{\tau}, t_{m}}^{c}}\left\|Y_{t_{m}}^{M, N}\right\|_{V}^{p}\right]$. Owing to the boundedness of the semigroup $E(t)$ in $V$, i.e., $\|E(t) \phi\|_{V} \leq\|\phi\|_{V}$, (3.9) with $\gamma=0$ and the structure of the taming scheme, i.e. $\frac{z}{1+\tau z} \leq \tau^{-1}$ for $z \geq 0$, one can infer

$$
\begin{aligned}
\left\|Y_{t_{m}}^{M, N}\right\|_{V} & \leq\left\|E\left(t_{m}\right) P_{N} X_{0}\right\|_{V}+\left\|P_{N} \mathscr{O}_{t_{m}}\right\|_{V}+\int_{0}^{t_{m}}\left\|E\left(t_{m}-s\right) \frac{P_{N} F\left(Y_{[s]}^{M, N}\right)}{1+\tau\left\|P_{N} F\left(Y_{\lfloor s]}^{M, N}\right)\right\|}\right\|_{V} \mathrm{~d} s \\
& \leq\left\|P_{N} X_{0}\right\|_{V}+\int_{0}^{t_{m}}\left(t_{m}-s\right)^{-\frac{1}{4}} \frac{\left\|P_{N} F\left(Y_{\lfloor s]}^{M, N}\right)\right\|}{1+\tau\left\|P_{N} F\left(Y_{\lfloor s\rfloor}^{M,}\right)\right\|} \mathrm{d} s+\left\|P_{N} \mathscr{O}_{t_{m}}\right\|_{V} \\
& \leq\left\|P_{N} X_{0}\right\|_{V}+\frac{4}{3} t_{m}^{\frac{3}{4}} \tau^{-1}+\left\|P_{N} \mathscr{O}_{t_{m}}\right\|_{V}, \quad m \in\{0,1,2, \ldots, M\} .
\end{aligned}
$$

Thanks to Lemma 3.1 and Assumption 4.3, we can show that for any $p \geq 2$,

$$
\left\|Y_{t_{m}}^{M, N}\right\|_{L^{p}(\Omega, V)} \leq C\left(1+\tau^{-1}\right), \quad m \in\{0,1,2, \ldots, M\} .
$$

Meanwhile, one can learn that

$$
\Omega_{R^{\tau}, t_{m}}^{c}=\Omega_{R^{\tau}, t_{m-1}}^{c}+\Omega_{R^{\tau}, t_{m-1}} \cdot\left\{\omega \in \Omega:\left\|Y_{t_{m}}^{M, N}\right\|_{V}>R^{\tau}\right\},
$$

and as a result

$$
\mathbb{1}_{\Omega_{R^{\tau}, t_{m}}^{c}}=\mathbb{1}_{\Omega_{R^{\tau}, t_{m-1}^{c}}^{c}}+\mathbb{1}_{\Omega_{R^{\tau}, t_{m-1}}} \cdot \mathbb{1}_{\left\{\left\|Y_{t_{m}}^{M, N}\right\|_{V}>R^{\tau}\right\}}=\sum_{i=0}^{m} \mathbb{1}_{\Omega_{R^{\tau}, t_{i-1}}} \cdot \mathbb{1}_{\left\{\left\|Y_{t_{i}}^{M, N}\right\|_{V}>R^{\tau}\right\}}
$$

where we recall $\mathbb{1}_{\Omega_{R}^{c} \tau_{-1}}=0$. By (4.46) and the Chebyshev inequality, one can show, for any $m \in$ 
$\{0,1, \ldots, M\}, M \in \mathbb{N}$

$$
\begin{aligned}
& \mathbb{E}\left[\mathbb{1}_{\Omega_{R^{\tau}, t_{m}}^{c}}\left\|Y_{t_{m}}^{M, N}\right\|_{V}^{p}\right]=\sum_{i=0}^{m} \mathbb{E}\left[\left\|Y_{t_{m}}^{M, N}\right\|_{V}^{p} \cdot \mathbb{1}_{\Omega_{R^{\tau}, t_{i-1}}} \mathbb{1}_{\left\{\left\|Y_{t_{i}}^{M, N}\right\|_{V}>R^{\tau}\right\}}\right] \\
& \leq \sum_{i=0}^{m}\left(\mathbb{E}\left[\left\|Y_{t_{m}}^{M, N}\right\|_{V}^{2 p}\right]\right)^{\frac{1}{2}}\left(\mathbb{E}\left[\mathbb{1}_{\Omega_{R^{\tau}, t_{i-1}}} \mathbb{1}_{\left\{\left\|Y_{t_{i}}^{M, N}\right\|_{V}>R^{\tau}\right\}}\right]\right)^{\frac{1}{2}} \\
& \leq \sum_{i=0}^{m} C\left(1+\tau^{-p}\right)\left(\mathbb{P}\left(\omega \in \Omega_{R^{\tau}, t_{i-1}}:\left\|Y_{t_{i}}^{M, N}\right\|_{V}>R^{\tau}\right)\right)^{\frac{1}{2}} \\
& =C\left(1+\tau^{-p}\right) \sum_{i=0}^{m}\left(\mathbb{P}\left(\omega \in \Omega: \mathbb{1}_{\Omega_{R} \tau, t_{i-1}}\left\|Y_{t_{i}}^{M, N}\right\|_{V}>R^{\tau}\right)\right)^{\frac{1}{2}} \\
& \leq C\left(1+\tau^{-p}\right) \sum_{i=0}^{m}\left(\mathbb{E}\left[\mathbb{1}_{\Omega_{R^{\tau}, t_{i-1}}}\left\|Y_{t_{i}}^{M, N}\right\|_{V}^{\frac{8(p+1)}{\beta}} /\left(R^{\tau}\right)^{\frac{8(p+1)}{\beta}}\right]\right)^{\frac{1}{2}} \\
& \leq C\left(1+\tau^{-p}\right) \sum_{i=0}^{m} \tau^{p+1}\left(\mathbb{E}\left[\mathbb{1}_{\Omega_{R} \tau, t_{i-1}}\left\|Y_{t_{i}}^{M, N}\right\|_{V}^{\frac{8(p+1)}{\beta}}\right]\right)^{\frac{1}{2}}<\infty .
\end{aligned}
$$

This estimate together with (4.42) yields the required estimate (4.44).

With Theorem 4.6 at hand, one can use standard arguments to obtain the coming corollaries.

Corollary 4.7. Let Assumptions 2.1-2.4, 4.3 be fulfilled and let the approximation process $Y_{t}^{M, N}, t \in$ $[0, T]$ be given by (4.19). Then for any $p \in[2, \infty)$ and $\beta<\frac{1}{2}$ we have

$$
\sup _{M, N \in \mathbb{N}, t \in[0, T]}\left\|Y_{t}^{M, N}\right\|_{L^{p}\left(\Omega, \dot{H}^{\beta}\right)}+\sup _{M, N \in \mathbb{N}, t \in[0, T]}\left\|Y_{t}^{M, N}\right\|_{L^{p}(\Omega, V)}<\infty .
$$

Corollary 4.8. Let Assumptions 2.1-2.4, 4.3 be fulfilled and let the approximation process $Y_{t}^{M, N}, t \in$ $[0, T]$ be given by (4.19). Then for any $p \in[2, \infty)$ and $\beta<\frac{1}{2}$ there exists a constant $C$, depending on $p, \beta, T$ and the initial data but not depending on $M, N$, such that

$$
\left\|Y_{t}^{M, N}-Y_{s}^{M, N}\right\|_{L^{p}(\Omega, H)} \leq C(t-s)^{\frac{\beta}{2}}, \quad 0 \leq s<t \leq T .
$$

\subsection{Refined temporal Hölder regularity results in negative Sobolev spaces}

In addition to the a priori moment bounds for the approximations, we further rely on refined temporal Hölder regularity results in negative Sobolev spaces, as stated in Corollary 4.11, which are essentially used in proving the temporal convergence rates of order almost $\frac{1}{2}$. To arrive at Corollary 4.11 , we also need commutativity properties of the nonlinearity and the improved temporal Hölder regularity of the solution in negative Sobolev spaces, as presented in Lemma 4.9 and Lemma 4.10, respectively.

For the first step, we reveal commutativity properties of the nonlinearity in the forthcoming lemma.

Lemma 4.9. Let $F: L^{6}(D ; \mathbb{R}) \rightarrow H$ be a mapping determined by Assumption 2.2. Then for any $\beta \in\left(0, \frac{1}{2}\right)$ and $\eta>\frac{1}{2}$ there exists a constant $C$ depending on $\beta, \eta,\left\{a_{i}\right\}_{i=0}^{3}$ such that

$$
\left\|F^{\prime}(\chi) v\right\|_{-\eta} \leq C\left(1+\max \left\{\|\chi\|_{V},\|\chi\|_{\beta}\right\}^{2}\right)\|v\|_{-\beta}, \quad \forall \chi \in V \cap \dot{H}^{\beta}, v \in V .
$$

Proof of Lemma 4.9. As $\beta \in\left(0, \frac{1}{2}\right)$, standard arguments with the Sobolev-Slobodeckij norm (see, 
e.g., $[46,(19.14)])$ and properties of the nonlinear mapping guarantee

$$
\begin{aligned}
\left\|F^{\prime}(\chi) \psi\right\|_{\beta}^{2} \leq & C\left\|F^{\prime}(\chi) \psi\right\|^{2}+C \int_{0}^{1} \int_{0}^{1} \frac{\left|f^{\prime}(\chi(x)) \psi(x)-f^{\prime}(\chi(y)) \psi(y)\right|^{2}}{|x-y|^{2 \beta+1}} \mathrm{~d} y \mathrm{~d} x \\
\leq & C\left\|F^{\prime}(\chi) \psi\right\|^{2}+C \int_{0}^{1} \int_{0}^{1} \frac{\left|f^{\prime}(\chi(x))(\psi(x)-\psi(y))\right|^{2}}{|x-y|^{2 \beta+1}} \mathrm{~d} y \mathrm{~d} x \\
& +C \int_{0}^{1} \int_{0}^{1} \frac{\left|\left[f^{\prime}(\chi(x))-f^{\prime}(\chi(y))\right] \psi(y)\right|^{2}}{|x-y|^{2 \beta+1}} \mathrm{~d} y \mathrm{~d} x \\
\leq & C\left\|F^{\prime}(\chi) \psi\right\|^{2}+C\left\|f^{\prime}(\chi(\cdot))\right\|_{V}^{2} \cdot\|\psi\|_{W^{\beta, 2}}^{2}+C\left\|f^{\prime \prime}(\chi(\cdot))\right\|_{V}^{2} \cdot\|\psi\|_{V}^{2} \cdot\|\chi\|_{W^{\beta, 2}}^{2} \\
\leq & C\left(1+\|\chi\|_{V}^{4}\right)\|\psi\|^{2}+C\left(1+\|\chi\|_{V}^{4}\right)\|\psi\|_{\beta}^{2}+C\left(1+\|\chi\|_{V}^{2}\right)\|\psi\|_{V}^{2} \cdot\|\chi\|_{\beta}^{2} \\
\leq & C\left(1+\max \left\{\|\chi\|_{V},\|\chi\|_{\beta}\right\}^{4}\right)\left(\|\psi\|_{\beta}^{2}+\|\psi\|_{V}^{2}\right) .
\end{aligned}
$$

Accordingly, for any $\beta \in\left(0, \frac{1}{2}\right)$ and $\eta>\frac{1}{2}$, one uses the Sobolev embedding inequality to derive

$$
\begin{aligned}
\left\|F^{\prime}(\chi) v\right\|_{-\eta} & =\sup _{\|\varphi\| \leq 1}\left|\left\langle A^{-\frac{\eta}{2}} F^{\prime}(\chi) v, \varphi\right\rangle\right|=\sup _{\|\varphi\| \leq 1}\left|\left\langle v,\left(F^{\prime}(\chi)\right)^{*} \cdot A^{-\frac{\eta}{2}} \varphi\right\rangle\right| \\
& =\sup _{\|\varphi\| \leq 1}\left|\left\langle A^{-\frac{\beta}{2}} v, A^{\frac{\beta}{2}} F^{\prime}(\chi) A^{-\frac{\eta}{2}} \varphi\right\rangle\right| \\
& \leq \sup _{\|\varphi\| \leq 1}\|v\|_{-\beta} \cdot\left\|F^{\prime}(\chi) A^{-\frac{\eta}{2}} \varphi\right\|_{\beta} \\
& \leq \sup _{\|\varphi\| \leq 1}\|v\|_{-\beta} \cdot C\left(1+\max \left\{\|\chi\|_{V},\|\chi\|_{\beta}\right\}^{2}\right)\left(\|\varphi\|_{\beta-\eta}+\left\|A^{-\frac{\eta}{2}} \varphi\right\|_{V}\right) \\
& \leq C_{\beta}\left(1+\max \left\{\|\chi\|_{V},\|\chi\|_{\beta}\right\}^{2}\right)\|v\|_{-\beta} .
\end{aligned}
$$

This completes the proof.

For the second step, we establish the improved temporal Hölder regularity of the solution in negative Sobolev spaces as follows.

Lemma 4.10. Let Assumptions 2.1-2.4, 4.3 be fulfilled and let the approximation process $Y_{t}^{M, N}, t \in[0, T]$ be produced by (4.19). Then for any $p \in[2, \infty), \delta \in\left[0, \frac{1}{2}\right]$ and $\beta<\frac{1}{2}$ there exists a constant $C$ depending on $T, p, \delta, \beta,\left\{a_{i}\right\}_{i=0}^{3}$ and the initial data but independent of $M, N$ such that

$$
\left\|Y_{t}^{M, N}-Y_{s}^{M, N}\right\|_{L^{p}\left(\Omega, \dot{H}^{-\delta}\right)} \leq C(t-s)^{\frac{\beta+\delta}{2}}, \quad 0 \leq s<t \leq T .
$$

Proof of Lemma 4.10. The definition (4.19) implies, for $0 \leq s<t \leq T$,

$$
\begin{aligned}
Y_{t}^{M, N}-Y_{s}^{M, N}= & \left(E_{N}(t-s)-I\right) Y_{s}^{M, N} \\
& +\int_{s}^{t} E_{N}(t-r) \frac{F_{N}\left(Y_{[r]}^{M, N}\right)}{1+\tau\left\|F_{N}\left(Y_{[r]}^{M, N}\right)\right\|} \mathrm{d} r+\int_{s}^{t} E_{N}(t-r) P_{N} \mathrm{~d} W(r) .
\end{aligned}
$$

Making use of (2.2), (2.4) and the inequality $\left\|\Gamma \Gamma_{1}\right\|_{\mathscr{L}_{2}} \leq\|\Gamma\|_{\mathscr{L}}\left\|\Gamma_{1}\right\|_{\mathscr{L}_{2}}, \Gamma \in \mathscr{L}(H), \Gamma_{1} \in \mathscr{L}_{2}(H)$ gives

$$
\left\|\int_{s}^{t} E_{N}(t-r) P_{N} \mathrm{~d} W(r)\right\|_{L^{p}\left(\Omega, \dot{H}^{-\delta}\right)} \leq C\left(\int_{s}^{t}\left\|A^{-\frac{\delta}{2}} E_{N}(t-r) P_{N}\right\|_{\mathscr{L}_{2}(H)}^{2} \mathrm{~d} r\right)^{\frac{1}{2}} \leq C(t-s)^{\frac{\beta+\delta}{2}} .
$$

A combination of (2.2) and Corollary 4.7 shows

$$
\begin{aligned}
\left\|\left(E_{N}(t-s)-I\right) Y_{s}^{M, N}\right\|_{L^{p}\left(\Omega, \dot{H}^{-\delta}\right)} & \leq\left\|A^{-\frac{\beta+\delta}{2}}(E(t-s)-I)\right\|_{\mathscr{L}(H)} \cdot\left\|Y_{s}^{M, N}\right\|_{L^{p}\left(\Omega, \dot{H}^{\beta}\right)} \\
& \leq C(t-s)^{\frac{\beta+\delta}{2}} .
\end{aligned}
$$


Now we proceed to estimate the remaining term in (4.56), with the help of (4.44) and (2.5),

$$
\left\|\int_{s}^{t} E(t-r) \frac{F_{N}\left(Y_{[r}^{M, N}\right)}{1+\tau\left\|F_{N}\left(Y_{[r]}^{M, N}\right)\right\|} d r\right\|_{L^{p}\left(\Omega, \dot{H}^{-\delta}\right)} \leq C \int_{s}^{t}\left\|F\left(Y_{\lfloor r\rfloor}^{M, N}\right)\right\|_{L^{p}(\Omega, H)} \mathrm{d} r \leq C(t-s) .
$$

Gathering (4.57), (4.58) and (4.59) we deduce from (4.56) that (4.55) is true.

It is worthwhile to mention that the approximate solution (4.19) enjoys higher order temporal Hölder regularity in negative Sobolev spaces $\dot{H}^{\delta}, \delta>0$, as indicated by Lemma 4.10. More precisely, the order of temporal Hölder regularity is increased to be $\frac{\beta+\delta}{2}$ when measured in the negative Sobolev space $\dot{H}^{\delta}, \delta \in\left(0, \frac{1}{2}\right]$, in contrast to order $\frac{\beta}{2}$ in $H$ by Corollary 4.8. So taking $\delta=0$ in (4.55) reduces into the usual temporal Hölder regularity results in $H$, i.e., (4.51) in Corollary 4.8.

Combining Lemmas 4.9, 4.10 with Corollary 4.7, one can easily arrive at the following corollary, which is used several times in the error analysis, to improve the strong convergence rate of the scheme.

Corollary 4.11. Let Assumptions 2.1-2.4, 4.3 be fulfilled and let the approximation process $Y_{t}^{M, N}, t \in$ $[0, T]$ be produced by (4.19). Then for any $p \in[2, \infty), \beta<\frac{1}{2}$ and $\eta>\frac{1}{2}$, there exists a constant $C$ depending on $T, p, \eta, \beta,\left\{a_{i}\right\}_{i=0}^{3}$ and the initial data but independent of $M, N$ such that

$$
\left\|F\left(Y_{t}^{M, N}\right)-F\left(Y_{s}^{M, N}\right)\right\|_{L^{p}\left(\Omega, \dot{H}^{-\eta}\right)} \leq C(t-s)^{\beta}, \quad 0<s<t<T .
$$

\subsection{Main results: error bounds for the full discretization}

At the moment, we are well prepared to prove the expected strong convergence rate of the proposed scheme, as stated in the following theorem.

Theorem 4.12 (The space-time full error bounds). Let Assumptions 2.1-2.4, 4.3 hold. Let $X(t)$ and $Y_{t}^{M, N}, t \in[0, T]$ be defined by (2.13) and (4.19), respectively. For any $p \in[2, \infty)$ and $\beta \in\left(0, \frac{1}{2}\right)$, there exists a constant $C$ depending on $T, p, \beta,\left\{a_{i}\right\}_{i=0}^{3}$ and the initial data but independent of $M, N$ such that

$$
\sup _{t \in[0, T]}\left\|X(t)-Y_{t}^{M, N}\right\|_{L^{p}(\Omega ; H)} \leq C\left(N^{-\beta}+\tau^{\beta}\right) .
$$

Proof of Theorem 4.12. Denoting $e_{t}^{M, N}:=P_{N} X(t)-Y_{t}^{M, N}$, we note that

$$
\left\|X(t)-Y_{t}^{M, N}\right\|_{L^{p}(\Omega ; H)} \leq\left\|\left(I-P_{N}\right) X(t)\right\|_{L^{p}(\Omega ; H)}+\left\|e_{t}^{M, N}\right\|_{L^{p}(\Omega ; H)},
$$

where

$$
\frac{\mathrm{d}}{\mathrm{d} t} e_{t}^{M, N}=-A_{N} e_{t}^{M, N}+F_{N}(X(t))-\frac{F_{N}\left(Y_{[t]}^{M, N}\right)}{1+\tau\left\|F_{N}\left(Y_{[t]}^{M, N}\right)\right\|} .
$$

This in conjunction with (2.5) tells us that

$$
\begin{aligned}
\left\|e_{t}^{M, N}\right\|^{p}= & p \int_{0}^{t}\left\|e_{s}^{M, N}\right\|^{p-2}\left\langle e_{s}^{M, N},-A_{N} e_{s}^{M, N}+F_{N}(X(s))-\frac{F_{N}\left(Y_{[s]}^{M, N}\right)}{1+\tau\left\|F_{N}\left(Y_{[s]}^{M, N}\right)\right\|}\right\rangle \mathrm{d} s \\
\leq & p \int_{0}^{t}\left\|e_{s}^{M, N}\right\|^{p-2}\left\langle e_{s}^{M, N}, F_{N}(X(s))-F_{N}\left(P_{N} X(s)\right)\right. \\
& \left.\quad+F_{N}\left(Y_{s}^{M, N}\right)-\frac{F_{N}\left(Y_{[s\rfloor}^{M, N}\right)}{1+\tau\left\|F_{N}\left(Y_{[s]}^{M, N}\right)\right\|}\right\rangle \mathrm{d} s+p L_{0} \int_{0}^{t}\left\|e_{s}^{M, N}\right\|^{p} \mathrm{~d} s \\
= & p L_{0} \int_{0}^{t}\left\|e_{s}^{M, N}\right\|^{p} \mathrm{~d} s+p \int_{0}^{t}\left\|e_{s}^{M, N}\right\|^{p-2}\left\langle e_{s}^{M, N}, F(X(s))-F\left(P_{N} X(s)\right)\right\rangle \mathrm{d} s \\
& +p \int_{0}^{t}\left\|e_{s}^{M, N}\right\|^{p-2}\left\langle e_{s}^{M, N}, F\left(Y_{s}^{M, N}\right)-F\left(Y_{\lfloor s\rfloor}^{M, N}\right)\right\rangle \mathrm{d} s \\
& +p \int_{0}^{t}\left\|e_{s}^{M, N}\right\|^{p-2}\left\langle e_{s}^{M, N}, \frac{\tau\left\|F_{N}\left(Y_{[s]}^{M, N}\right)\right\| \cdot F\left(Y_{[s]}^{M, N}\right)}{1+\tau\left\|F_{N}\left(Y_{[s]}^{M, N}\right)\right\|}\right\rangle \mathrm{d} s \\
= & : p L_{0} \int_{0}^{t}\left\|e_{s}^{M, N}\right\|^{p} \mathrm{~d} s+J_{0}+J_{1}+J_{2} .
\end{aligned}
$$


Following the same lines as in estimates of (3.16) and (3.17) one can bound the item $J_{0}$ as follows,

$$
\begin{aligned}
\mathbb{E}\left[J_{0}\right] & \leq p \mathbb{E} \int_{0}^{t}\left\|e_{s}^{M, N}\right\|^{p-1}\left\|F(X(s))-F\left(P_{N} X(s)\right)\right\| \mathrm{d} s \\
& \leq(p-1) \int_{0}^{t} \mathbb{E}\left[\left\|e_{s}^{M, N}\right\|^{p}\right] \mathrm{d} s+\int_{0}^{t} \mathbb{E}\left[\left\|F(X(s))-F\left(P_{N} X(s)\right)\right\|^{p}\right] \mathrm{d} s \\
& \leq(p-1) \int_{0}^{t} \mathbb{E}\left[\left\|e_{s}^{M, N}\right\|^{p}\right] \mathrm{d} s+C\left(\frac{1}{N}\right)^{p \beta}
\end{aligned}
$$

The term $J_{2}$ is also easy to be treated, after taking the Hölder inequality and (4.44) into account:

$$
\begin{aligned}
\mathbb{E}\left[J_{2}\right] & \leq p \mathbb{E} \int_{0}^{t}\left\|e_{s}^{M, N}\right\|^{p-1} \cdot \tau\left\|F\left(Y_{\lfloor s\rfloor}^{M, N}\right)\right\|^{2} \mathrm{~d} s \\
& \leq(p-1) \int_{0}^{t} \mathbb{E}\left[\left\|e_{s}^{M, N}\right\|^{p}\right] \mathrm{d} s+\tau^{p} \int_{0}^{t} \mathbb{E}\left[\left\|F\left(Y_{\lfloor s\rfloor}^{M, N}\right)\right\|^{2 p}\right] \mathrm{d} s \\
& \leq(p-1) \int_{0}^{t} \mathbb{E}\left[\left\|e_{s}^{M, N}\right\|^{p}\right] \mathrm{d} s+C \tau^{p} .
\end{aligned}
$$

The remaining term $J_{1}$ must be handled more carefully. As usual, such a term is simply treated with the aid of temporal Hölder regularity of $Y_{s}^{M, N}$ together with the Cauchy-Schwarz inequality and Hölder's inequality, but to only attain order $\tau^{\frac{\beta}{2}}$. In our analysis, we decompose $P_{N} X(s)-Y_{s}^{M, N}$ in the inner product into three parts. To do so we recall that

$$
e_{s}^{M, N}=P_{N} X(s)-Y_{s}^{M, N}=\int_{0}^{s} E(s-r)\left(F_{N}(X(r))-\frac{F_{N}\left(Y_{[r]}^{M, N}\right)}{1+\tau\left\|F_{N}\left(Y_{[r]}^{M, N}\right)\right\|}\right) \mathrm{d} r
$$

and split $J_{1}$ into three terms:

$$
\begin{aligned}
J_{1}= & p \int_{0}^{t}\left\|e_{s}^{M, N}\right\|^{p-2}\left\langle\int_{0}^{s} E(s-r)\left(F_{N}(X(r))-\frac{F_{N}\left(Y_{\lfloor r\rfloor}^{M, N}\right)}{1+\tau\left\|F_{N}\left(Y_{\lfloor r\rfloor}^{M, N}\right)\right\|}\right) \mathrm{d} r, F\left(Y_{s}^{M, N}\right)-F\left(Y_{\lfloor s\rfloor}^{M, N}\right)\right\rangle \mathrm{d} s \\
= & p \int_{0}^{t}\left\|e_{s}^{M, N}\right\|^{p-2}\left\langle\int_{0}^{s} E(s-r)\left(F_{N}(X(r))-F_{N}\left(Y_{r}^{M, N}\right)\right) \mathrm{d} r, F\left(Y_{s}^{M, N}\right)-F\left(Y_{\lfloor s\rfloor}^{M, N}\right)\right\rangle \mathrm{d} s \\
& +p \int_{0}^{t}\left\|e_{s}^{M, N}\right\|^{p-2}\left\langle\int_{0}^{s} E(s-r)\left(F_{N}\left(Y_{r}^{M, N}\right)-F_{N}\left(Y_{\lfloor r\rfloor}^{M, N}\right)\right) \mathrm{d} r, F\left(Y_{s}^{M, N}\right)-F\left(Y_{\lfloor s\rfloor}^{M, N}\right)\right\rangle \mathrm{d} s \\
& +p \int_{0}^{t}\left\|e_{s}^{M, N}\right\|^{p-2}\left\langle\int_{0}^{s} E(s-r) \frac{\tau\left\|F_{N}\left(Y_{\lfloor r\rfloor}^{M, N}\right)\right\| \cdot F_{N}\left(Y_{[r]}^{M, N}\right)}{1+\tau\left\|F_{N}\left(Y_{\lfloor r\rfloor}^{M, N}\right)\right\|} \mathrm{d} r, F\left(Y_{s}^{M, N}\right)-F\left(Y_{\lfloor s\rfloor}^{M, N}\right)\right\rangle \mathrm{d} s \\
= & J_{11}+J_{12}+J_{13} .
\end{aligned}
$$

Since the estimates concerning $J_{11}$ and $J_{12}$ are demanding, we handle the item $J_{13}$ first. Utilizing (2.5), the Hölder inequality, (4.44) and (4.51) results in

$$
\begin{aligned}
\mathbb{E}\left[\left|J_{13}\right|\right] \leq & p \mathbb{E} \int_{0}^{t} \int_{0}^{s}\left\|e_{s}^{M, N}\right\|^{p-2} \cdot \tau\left\|F\left(Y_{\lfloor r\rfloor}^{M, N}\right)\right\|^{2} \cdot\left\|F\left(Y_{s}^{M, N}\right)-F\left(Y_{\lfloor s\rfloor}^{M, N}\right)\right\| \mathrm{d} r \mathrm{~d} s \\
\leq & C \tau \mathbb{E} \int_{0}^{t} \int_{0}^{s}\left\|e_{s}^{M, N}\right\|^{p-2}\left\|F\left(Y_{\lfloor r\rfloor}^{M, N}\right)\right\|^{2}\left(1+\left\|Y_{s}^{M, N}\right\|_{V}^{2}+\left\|Y_{\lfloor s\rfloor}^{M, N}\right\|_{V}^{2}\right)\left\|Y_{s}^{M, N}-Y_{\lfloor s\rfloor}^{M, N}\right\| \mathrm{d} r \mathrm{~d} s \\
\leq & C \int_{0}^{t} \mathbb{E}\left[\left\|e_{s}^{M, N}\right\|^{p}\right] \mathrm{d} s+C \tau^{\frac{p}{2}} \int_{0}^{t} \mathbb{E}\left[\left\lfloor\int _ { 0 } ^ { s } \| F ( Y _ { \lfloor r \rfloor } ^ { M , N } ) \| ^ { 2 } \cdot \left(1+\left\|Y_{s}^{M, N}\right\|_{V}^{2}\right.\right.\right. \\
& \left.\left.+\left\|Y_{\lfloor s\rfloor}^{M, N}\right\|_{V}^{2}\right)\left.\left\|Y_{s}^{M, N}-Y_{\lfloor s\rfloor}^{M, N}\right\| \mathrm{d} r\right|^{\frac{p}{2}}\right] \mathrm{d} s \\
\leq & C \int_{0}^{t} \mathbb{E}\left[\left\|e_{s}^{M, N}\right\|^{p}\right] \mathrm{d} s+C \tau^{\frac{p}{2}\left(1+\frac{\beta}{2}\right)}
\end{aligned}
$$


At the moment we come to the estimate of $J_{11}$ and use the Taylor formula, the self-adjointness of operators $F^{\prime}(u)$ and $P_{N}$ to infer that

$$
\begin{aligned}
& \mathbb{E}\left[\left|J_{11}\right|\right]= p \mathbb{E}\left[\left|\int_{0}^{t}\left\|e_{s}^{M, N}\right\|^{p-2}\left\langle\int_{0}^{s} E(s-r)\left(F_{N}(X(r))-F_{N}\left(Y_{r}^{M, N}\right)\right) \mathrm{d} r, F\left(Y_{s}^{M, N}\right)-F\left(Y_{\lfloor s\rfloor}^{M, N}\right)\right\rangle \mathrm{d} s\right|\right] \\
&= p \mathbb{E}\left[\mid \int_{0}^{t}\left\|e_{s}^{M, N}\right\|^{p-2}\left\langle\int_{0}^{s} E(s-r) P_{N} \int_{0}^{1} F^{\prime}\left(Y_{r}^{M, N}+\sigma\left(X(r)-Y_{r}^{M, N}\right)\right) \mathrm{d} \sigma\right.\right. \\
&\left.\left.\cdot\left(X(r)-Y_{r}^{M, N}\right) \mathrm{d} r, F\left(Y_{s}^{M, N}\right)-F\left(Y_{\lfloor s\rfloor}^{M, N}\right)\right\rangle \mathrm{d} s \mid\right] \\
&=p \mathbb{E}\left[\mid \int_{0}^{t} \int_{0}^{s} \int_{0}^{1}\left\|e_{s}^{M, N}\right\|^{p-2}\left\langle X(r)-Y_{r}^{M, N},\left(F^{\prime}\left(Y_{r}^{M, N}+\sigma\left(X(r)-Y_{r}^{M, N}\right)\right)\right)^{*}\right.\right. \\
&\left.\left.P_{N} E(s-r)\left[F\left(Y_{s}^{M, N}\right)-F\left(Y_{\lfloor s\rfloor}^{M, N}\right)\right]\right\rangle \mathrm{d} \sigma \mathrm{d} r \mathrm{~d} s \mid\right] \\
& \leq p \mathbb{E} \int_{0}^{t} \int_{0}^{s} \int_{0}^{1}\left\|e_{s}^{M, N}\right\|^{p-2} \cdot\left\|X(r)-Y_{r}^{M, N}\right\| \cdot \| F^{\prime}\left(Y_{r}^{M, N}+\sigma\left(X(r)-Y_{r}^{M, N}\right)\right) \\
& P_{N} A^{\frac{\eta}{2}} E(s-r) A^{-\frac{\eta}{2}}\left[F\left(Y_{s}^{M, N}\right)-F\left(Y_{\lfloor s\rfloor}^{M, N}\right)\right] \| \mathrm{d} \sigma \mathrm{d} r \mathrm{~d} s \\
& \leq C \mathbb{E} \int_{0}^{t} \int_{0}^{s}\left\|e_{s}^{M, N}\right\|^{p-2}\left\|X(r)-Y_{r}^{M, N}\right\| \cdot\left(1+\|X(r)\|_{V}^{2}+\left\|Y_{r}^{M, N}\right\|_{V}^{2}\right) \\
& \times(s-r)^{-\frac{\eta}{2}}\left\|A^{-\frac{\eta}{2}}\left[F\left(Y_{s}^{M, N}\right)-F\left(Y_{\lfloor s\rfloor}^{M, N}\right)\right]\right\| \mathrm{d} r \mathrm{~d} s .
\end{aligned}
$$

Further, employing the Young inequalities $a b \leq \frac{a^{\delta}}{\delta}+\frac{b^{\delta^{\prime}}}{\delta^{\prime}}$ for $a, b \geq 0, \delta, \delta^{\prime}>1, \frac{1}{\delta}+\frac{1}{\delta^{\prime}}=1$, the Hölder inequality, the transformation of integral domain and taking $\frac{1}{2}<\eta<1$ give

$$
\begin{aligned}
\mathbb{E}\left[\left|J_{11}\right|\right] \leq & C \mathbb{E} \int_{0}^{t} \int_{0}^{s}(s-r)^{-\frac{\eta}{2}}\left\|e_{s}^{M, N}\right\|^{p} \mathrm{~d} r \mathrm{~d} s+C \mathbb{E} \int_{0}^{t} \int_{0}^{s}(s-r)^{-\frac{\eta}{2}}\left\|X(r)-Y_{r}^{M, N}\right\|^{\frac{p}{2}} \\
& \times\left(1+\|X(r)\|_{V}^{p}+\left\|Y_{r}^{M, N}\right\|_{V}^{p}\right)\left\|A^{-\frac{\eta}{2}}\left[F\left(Y_{s}^{M, N}\right)-F\left(Y_{\lfloor s\rfloor}^{M, N}\right)\right]\right\|^{\frac{p}{2}} \mathrm{~d} r \mathrm{~d} s \\
\leq & C \int_{0}^{t} \mathbb{E}\left[\left\|e_{s}^{M, N}\right\|^{p}\right] \mathrm{d} s+C \mathbb{E} \int_{0}^{t} \int_{0}^{s}(s-r)^{-\frac{\eta}{2}}\left\|X(r)-Y_{r}^{M, N}\right\|^{p} \mathrm{~d} r \mathrm{~d} s \\
& +C \mathbb{E} \int_{0}^{t} \int_{0}^{s}(s-r)^{-\frac{\eta}{2}}\left(1+\|X(r)\|_{V}^{2 p}+\left\|Y_{r}^{M, N}\right\|_{V}^{2 p}\right)\left\|A^{-\frac{\eta}{2}}\left[F\left(Y_{s}^{M, N}\right)-F\left(Y_{\lfloor s\rfloor}^{M, N}\right)\right]\right\|^{p} \mathrm{~d} r \mathrm{~d} s \\
\leq & C \int_{0}^{t} \mathbb{E}\left[\left\|e_{s}^{M, N}\right\|^{p}\right] \mathrm{d} s+C \int_{0}^{t} \mathbb{E}\left[\left\|X(s)-Y_{s}^{M, N}\right\|^{p}\right] \mathrm{d} s \\
& +C \int_{0}^{t} \int_{0}^{s}(s-r)^{-\frac{\eta}{2}} \mathbb{E}\left[\left(1+\|X(r)\|_{V}^{2 p}+\left\|Y_{r}^{M, N}\right\|_{V}^{2 p}\right)\left\|A^{-\frac{\eta}{2}}\left[F\left(Y_{s}^{M, N}\right)-F\left(Y_{\lfloor s\rfloor}^{M, N}\right)\right]\right\|^{p}\right] \mathrm{d} r \mathrm{~d} s,
\end{aligned}
$$

where the first step is trivial for $p=2$ due to (4.70) and holds true for $p>2$ after using the aforementioned Young inequality with $\delta=\frac{p}{p-2}, \delta^{\prime}=\frac{p}{2}$. To proceed further, we resort to Corollary 4.11 as well as (2.14), (4.44) and achieve

$$
\begin{aligned}
\mathbb{E}\left[\left|J_{11}\right|\right] & \leq C \int_{0}^{t} \mathbb{E}\left[\left\|e_{s}^{M, N}\right\|^{p}\right] \mathrm{d} s+C \lambda_{N+1}^{-\frac{p \beta}{2}}+C \int_{0}^{t}\left(\mathbb{E}\left[\left\|A^{-\frac{\eta}{2}}\left[F\left(Y_{s}^{M, N}\right)-F\left(Y_{\lfloor s\rfloor}^{M, N}\right)\right]\right\|^{2 p}\right]\right)^{\frac{1}{2}} \mathrm{~d} s \\
& \leq C \int_{0}^{t} \mathbb{E}\left[\left\|e_{s}^{M, N}\right\|^{p}\right] \mathrm{d} s+C\left(\frac{1}{N}\right)^{p \beta}+C \tau^{p \beta} .
\end{aligned}
$$

Finally, it remains to deal with the estimate of $J_{12}$. By the Young inequalities $a b \leq \frac{a^{\delta}}{\delta}+\frac{b^{\delta^{\prime}}}{\delta^{\prime}}$ for $a, b \geq 0$, 
$\delta, \delta^{\prime}>1, \frac{1}{\delta}+\frac{1}{\delta^{\prime}}=1$ and putting $\frac{1}{2}<\eta<1$ one can derive that

$$
\begin{aligned}
\mathbb{E}\left[\left|J_{12}\right|\right]= & p \mathbb{E}\left[\mid \int_{0}^{t}\left\|e_{s}^{M, N}\right\|^{p-2}\left\langle\int_{0}^{s} A^{\eta} E(s-r) A^{-\frac{\eta}{2}}\left(F_{N}\left(Y_{r}^{M, N}\right)-F_{N}\left(Y_{\lfloor r\rfloor}^{M, N}\right)\right) \mathrm{d} r,\right.\right. \\
& \left.\left.A^{-\frac{\eta}{2}}\left(F_{N}\left(Y_{s}^{M, N}\right)-F_{N}\left(Y_{\lfloor s\rfloor}^{M, N}\right)\right)\right\rangle \mathrm{d} s \mid\right] \\
\leq & C \mathbb{E} \int_{0}^{t} \int_{0}^{s}(s-r)^{-\eta}\left\|e_{s}^{M, N}\right\|^{p-2} \cdot\left\|A^{-\frac{\eta}{2}}\left(F\left(Y_{r}^{M, N}\right)-F\left(Y_{\lfloor r\rfloor}^{M, N}\right)\right)\right\| \\
& \times\left\|A^{-\frac{\eta}{2}}\left(F\left(Y_{s}^{M, N}\right)-F\left(Y_{\lfloor s\rfloor}^{M, N}\right)\right)\right\| \mathrm{d} r \mathrm{~d} s \\
\leq & C \int_{0}^{t} \int_{0}^{s}(s-r)^{-\eta} \mathbb{E}\left[\left\|e_{s}^{M, N}\right\|^{p}\right] \mathrm{d} r \mathrm{~d} s+C \mathbb{E} \int_{0}^{t} \int_{0}^{s}(s-r)^{-\eta}\left\|A^{-\frac{\eta}{2}}\left(F\left(Y_{r}^{M, N}\right)-F\left(Y_{\lfloor r\rfloor}^{M, N}\right)\right)\right\|^{\frac{p}{2}} \\
\leq & C \int_{0}^{t} \mathbb{E}\left[\left\|A^{-\frac{\eta}{2}}\left(F\left(Y_{s}^{M, N}\right)-F\left(Y_{\lfloor s\rfloor}^{M, N}\right)\right)\right\|^{\frac{p}{2}} \mathrm{~d} r \mathrm{~d} s\right. \\
& +C \int_{0}^{t} \int_{0}^{s}(s-r)^{-\eta} \mathbb{d} s+C \int_{0}^{t} \int_{0}^{s}(s-r)^{-\eta} \mathbb{E}\left[\left\|A^{-\frac{\eta}{2}}\left(F\left(Y_{s}^{M, N}\right)-F\left(Y_{\lfloor s\rfloor}^{M, N}\right)\right)\right\|^{p}\right] \mathrm{d} r \mathrm{~d} s,
\end{aligned}
$$

where the last step but one is trivial for $p=2$ and holds true for $p>2$ after using the aforementioned Young inequality with $\delta=\frac{p}{p-2}, \delta^{\prime}=\frac{p}{2}$. In the last step, we used the inequality $a b \leq \frac{a^{2}}{2}+\frac{b^{2}}{2}, a, b \geq 0$. Again, the use of Corollary 4.11 leads us to

$$
\mathbb{E}\left[\left|J_{12}\right|\right] \leq C \int_{0}^{t} \mathbb{E}\left[\left\|e_{s}^{M, N}\right\|^{p}\right] \mathrm{d} s+C \tau^{\beta p},
$$

which together with (4.69), (4.72) forces us to recognize from (4.68) that

$$
\mathbb{E}\left[\left|J_{1}\right|\right] \leq C \int_{0}^{t} \mathbb{E}\left[\left\|e_{s}^{M, N}\right\|^{p}\right] \mathrm{d} s+C\left(\frac{1}{N}\right)^{p \beta}+C \tau^{\beta p} .
$$

Plugging this and (4.65), (4.66) into (4.64), taking expectations and applying the Gronwall inequality give

$$
\left\|e_{t}^{M, N}\right\|_{L^{p}(\Omega ; H)} \leq C\left(N^{-\beta}+\tau^{\beta}\right) .
$$

Therefore, the desired error bound follows from (4.62) by taking (2.15) and (3.2) into account.

\section{Numerical experiments}

Some numerical experiments are performed in this section to test the previous theoretical findings. Let us consider the stochastic Allen-Cahn equation with additive space-time white noise, described by

$$
\left\{\begin{array}{l}
\frac{\partial u}{\partial t}=\frac{\partial^{2} u}{\partial x^{2}}+u-u^{3}+\dot{W}(t), \quad t \in(0,1], x \in(0,1), \\
u(0, x)=\sin (\pi x), \quad x \in(0,1) \\
u(t, 0)=u(t, 1)=0, \quad t \in(0,1]
\end{array}\right.
$$

Here $\{W(t)\}_{t \in[0, T]}$ is a cylindrical $I$-Wiener process represented by (2.7). In what follows, we will use the new fully discrete scheme (4.1) to approximate the continuous problem (5.1). Error bounds are always measured in terms of mean-square approximation errors at the endpoint $T=1$, caused by spatial and temporal discretizations and the expectations are approximated by computing averages over 1000 samples.

Before proceeding further with simulations, it is helpful to mention that the stochastic convolution in the scheme (5.1) is easily implementable once one realizes that $\int_{t_{m}}^{t_{m+1}} E_{N}\left(t_{m+1}-s\right) P_{N} \mathrm{~d} W(s)=\sum_{i=1}^{N} \Lambda_{i} e_{i}$, 
where $\Lambda_{i}=\int_{t_{m}}^{t_{m+1}} e^{-\left(t_{m+1}-s\right) \lambda_{i}} \mathrm{~d} \beta(s), 1 \leq i \leq N$ are independent, zero-mean normally distributed random variables with explicit variances $\mathbb{E}\left[\left|\Lambda_{i}\right|^{2}\right]=\frac{1-e^{-2 \lambda_{i} \tau}}{2 \lambda_{i}}$. For more details on the implementation of so-called AEE schemes, one can consult [30, section 3] and [49, section 4.1].

To visually inspect the convergence rates in space, we identify the "exact" solution by using the full discretization with $M_{\text {exact }}=N_{\text {exact }}=2^{11}=2048$. The spatial approximation errors $\left\|X(1)-X^{N}(1)\right\|_{L^{2}(\Omega ; H)}$ with $N=2^{i}, i=2, \ldots, 7$ are depicted in Fig.1, against $\frac{1}{N}$ on a log-log scale, where one can observe that the resulting spatial errors decrease at a slope close to $1 / 2$. This is consistent with the previous theoretical result (3.13).

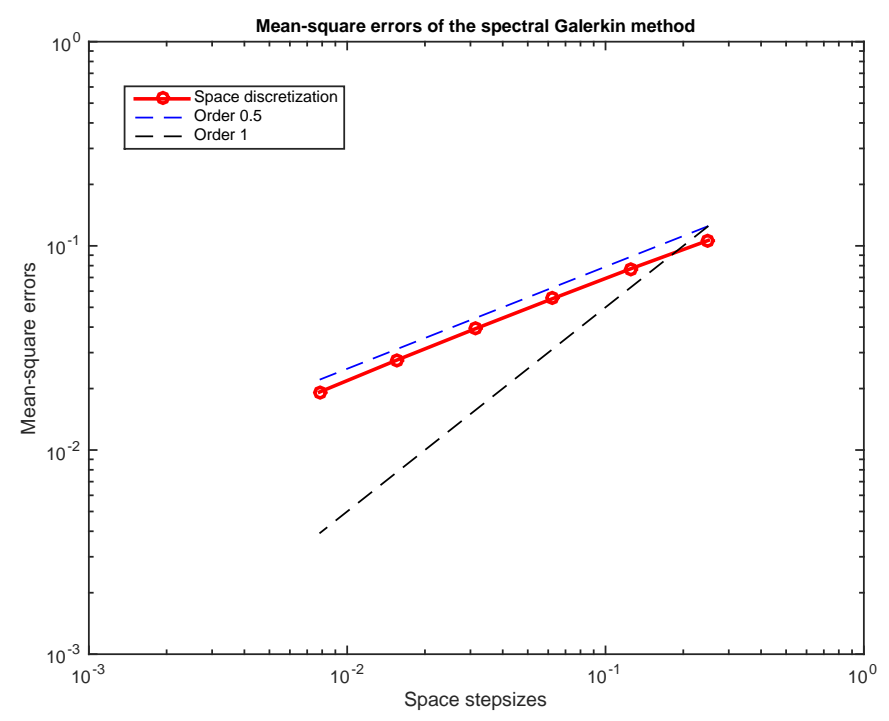

FIG 1. The convergence rate of the spectral Galerkin spatial discretization.

Moreover, we attempt to illustrate the error bound (4.61) for the fully discrete scheme (5.1). As implied by (4.61), the convergence rate in space is identical to that in time. Consequently, we take $M=N, p=2$ and $\beta=\frac{1}{2}-\varepsilon$ with arbitrarily small $\varepsilon>0$ in (4.61) to arrive at

$$
\left\|X(1)-Y_{t_{N}^{N, N}}^{N}\right\|_{L^{2}(\Omega ; H)} \leq C_{\varepsilon} N^{-\frac{1}{2}+\varepsilon} .
$$

To see (5.2), we, similarly as above, do a full discretization on a very fine mesh with $M_{\text {exact }}=N_{\text {exact }}=$ $2^{11}=2048$ to compute the "exact" solution. Six different mesh parameters $N=2^{i}, i=2,3, \ldots, 7$ are then used to get six full discretizations. The resulting errors are listed in Table 1 and plotted in Fig.2 on a $\log$-log scale. From Fig.2, one can observe the expected convergence rate of order almost $\frac{1}{2}$, which agrees with that indicated in (5.2).

TABLE 1

Computational errors of the fully discrete scheme with $M=N$

\begin{tabular}{cccccc}
\hline$N=2^{2}$ & $N=2^{3}$ & $N=2^{4}$ & $N=2^{5}$ & $N=2^{6}$ & $N=2^{7}$ \\
\hline 0.106381 & 0.077172 & 0.055174 & 0.039209 & 0.027624 & 0.019225 \\
\hline
\end{tabular}




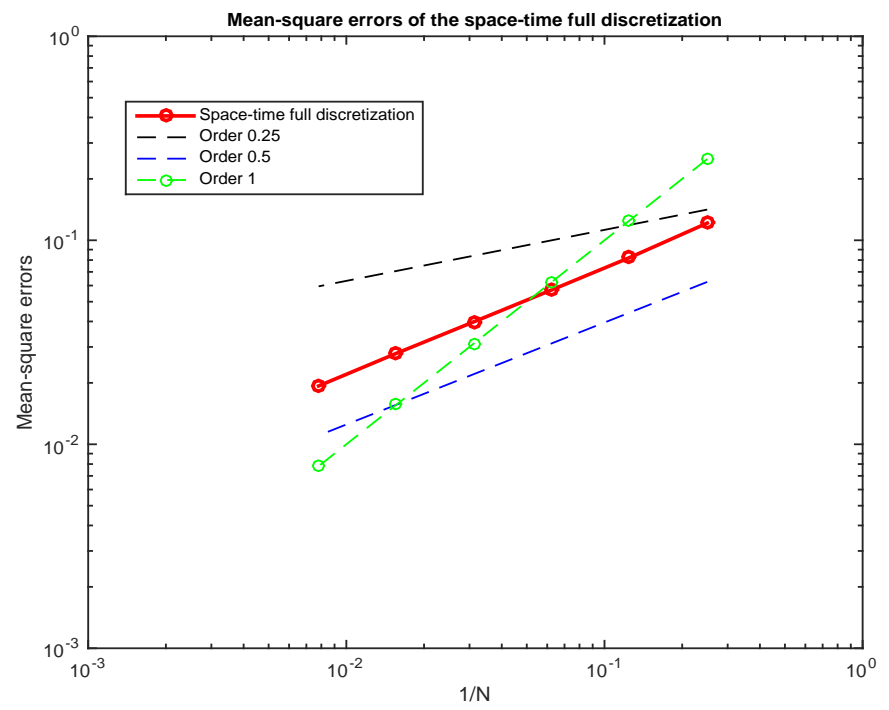

FIG 2. The convergence rate of the space-time full discretization.

\section{References}

[1] R. Anton, D. Cohen, and L. Quer-Sardanyons. A fully discrete approximation of the onedimensional stochastic heat equation. arXiv preprint arXiv:1711.08340, to appear in IMA Journal of Numerical Analysis, dry060, 2017.

[2] M. Beccari, M. Hutzenthaler, A. Jentzen, R. Kurniawan, F. Lindner, and D. Salimova. Strong and weak divergence of exponential and linear-implicit euler approximations for stochastic partial differential equations with superlinearly growing nonlinearities. arXiv preprint arXiv:1903.06066, 2019.

[3] S. Becker, B. Gess, A. Jentzen, and P. E. Kloeden. Strong convergence rates for explicit spacetime discrete numerical approximations of stochastic Allen-Cahn equations. arXiv preprint arXiv:1711.02423, 2017.

[4] S. Becker and A. Jentzen. Strong convergence rates for nonlinearity-truncated Euler-type approximations of stochastic Ginzburg-Landau equations. Stochastic Processes and their Applications, 129:28-69, 2019.

[5] D. Blomker and A. Jentzen. Galerkin approximations for the stochastic Burgers equation. SIAM Journal on Numerical Analysis, 51(1):694-715, 2013.

[6] C.-E. Bréhier, J. Cui, and J. Hong. Strong convergence rates of semi-discrete splitting approximations for stochastic Allen-Cahn equation. IMA Journal of Numerical Analysis, 39:2096-2134, 2019.

[7] C.-E. Bréhier and L. Goudenège. Weak convergence rates of splitting schemes for the stochastic Allen-Cahn equation. arXiv preprint arXiv:1804.04061, to appear in BIT Numerical Mathematics, 2018.

[8] C.-E. Bréhier and L. Goudenège. Analysis of some splitting schemes for the stochastic Allen-Cahn equation. Discrete Contin. Dyn. Syst. B, 24:4169-4190, 2019.

[9] Y. Cao, J. Hong, and Z. Liu. Approximating stochastic evolution equations with additive white and rough noises. SIAM Journal on Numerical Analysis, 55(4):1958-1981, 2017.

[10] S. Cerrai. Second order PDE's in finite and infinite dimension: a probabilistic approach, volume 1762. Springer Science \& Business Media, 2001.

[11] G. Da Prato. Kolmogorov Equations for Stochastic PDEs. Springer Science \& Business Media, 
2004.

[12] G. Da Prato and J. Zabczyk. Stochastic equations in infinite dimensions, volume 152. Cambridge university press, 2014.

[13] A. Davie and J. Gaines. Convergence of numerical schemes for the solution of parabolic stochastic partial differential equations. Mathematics of Computation, 70(233):121-134, 2001.

[14] Debussche A. Weak approximation of stochastic partial differential equations: the nonlinear case. Math. Comp., 80:89-117, 2011.

[15] Debussche A., Printems J. Weak order for the discretization of the stochastic heat equation. Math. Comp., 78:845-863, 2009.

[16] W. G. Faris and G. Jona-Lasinio. Large fluctuations for a nonlinear heat equation with noise. Journal of Physics A: Mathematical and General, 15(10):3025, 1982.

[17] X. Feng, Y. Li, and Y. Zhang. Finite element methods for the stochastic Allen-Cahn equation with gradient-type multiplicative noise. SIAM Journal on Numerical Analysis, 55(1):194-216, 2017.

[18] C. Gardiner. Handbook of stochastic methods for physics, chemistry and the natural sciences. Springer series in synergetics, 13:149-168, 1986.

[19] I. Gyöngy. Lattice approximations for stochastic quasi-linear parabolic partial differential equations driven by space-time white noise. i. Potential Anal., 9(1):1-25, 1998.

[20] I. Gyöngy, S. Sabanis, and D. Šiška. Convergence of tamed Euler schemes for a class of stochastic evolution equations. Stoch. PDE: Anal. Comp., 4(2):225-245, 2016.

[21] Gyöngy I. Lattice approximations for stochastic quasi-linear parabolic partial differential equations driven by space-time white noise ii. Potential Anal., 11(1):1-37, 1999.

[22] M. Hutzenthaler and A. Jentzen. On a perturbation theory and on strong convergence rates for stochastic ordinary and partial differential equations with non-globally monotone coefficients. arXiv preprint arXiv:1401.0295, 2014.

[23] M. Hutzenthaler and A. Jentzen. Numerical approximation of stochastic differential equations with non-globally Lipschitz continuous coefficients. Mem. Amer. Math. Soc., 236(1112), 2015.

[24] M. Hutzenthaler, A. Jentzen, and P. E. Kloeden. Strong and weak divergence in finite time of Euler's method for stochastic differential equations with non-globally Lipschitz continuous coefficients. Proceedings of the Royal Society of London A: Mathematical, Physical and Engineering Sciences, 467(2130):1563-1576, 2011.

[25] M. Hutzenthaler, A. Jentzen, and P. E. Kloeden. Strong convergence of an explicit numerical method for sdes with non-globally lipschitz continuous coefficients. Ann. Appl. Probab., 22(4):1611-1641, 2012.

[26] M. Hutzenthaler, A. Jentzen, and D. Salimova. Strong convergence of full-discrete nonlinearitytruncated accelerated exponential Euler-type approximations for stochastic Kuramoto-Sivashinsky equations. arXiv preprint arXiv:1604.02053, 2016.

[27] M. Hutzenthaler, A. Jentzen, and X. Wang. Exponential integrability properties of numerical approximation processes for nonlinear stochastic differential equations. Mathematics of Сотриtation, 87(311):1353-1413, 2018.

[28] A. Jentzen. Higher order pathwise numerical approximations of spdes with additive noise. SIAM Journal on Numerical Analysis, 49(2):642-667, 2011.

[29] A. Jentzen, P. Kloeden, and G. Winkel. Efficient simulation of nonlinear parabolic spdes with additive noise. The Annals of Applied Probability, 21(3):908-950, 2011.

[30] A. Jentzen and P. E. Kloeden. Overcoming the order barrier in the numerical approximation of stochastic partial differential equations with additive space-time noise. Proc. R. Soc. Lond. Ser. A Math. Phys. Eng. Sci., 465(2102):649-667, 2009.

[31] A. Jentzen and P. E. Kloeden. Taylor approximations for stochastic partial differential equations, volume 83. SIAM, 2011.

[32] A. Jentzen and P. Pušnik. Strong convergence rates for an explicit numerical approximation method for stochastic evolution equations with non-globally lipschitz continuous nonlinearities. arXiv preprint arXiv:1504.03523, 2015. 
[33] M. Kovács, S. Larsson, and F. Lindgren. On the backward Euler approximation of the stochastic Allen-Cahn equation. Journal of Applied Probability, 52(2):323-338, 2015.

[34] M. Kovács, S. Larsson, and F. Lindgren. On the discretisation in time of the stochastic Allen-Cahn equation. Mathematische Nachrichten, 291(5-6):966-995, 2018.

[35] R. Kruse. Strong and weak approximation of semilinear stochastic evolution equations. Springer, 2014.

[36] D. Liu. Convergence of the spectral method for stochastic Ginzburg-Landau equation driven by space-time white noise. Communications in Mathematical Sciences, 1(2):361-375, 2003.

[37] Z. Liu and Z. Qiao. Strong approximation of stochastic Allen-Cahn equation with white noise. arXiv preprint arXiv:1801.09348, 2018.

[38] G. J. Lord, C. E. Powell, and T. Shardlow. An Introduction to Computational Stochastic PDEs. Number 50. Cambridge University Press, 2014.

[39] Lord G.J., Tambue A. A modified semi-implicit euler-maruyama scheme for finite element discretization of spdes with additive noise. Applied Mathematics and Computation, 332:105-122, 2018.

[40] A. K. Majee and A. Prohl. Optimal strong rates of convergence for a space-time discretization of the stochastic Allen-Cahn equation with multiplicative noise. arXiv preprint arXiv:1705.09997, 2017.

[41] A. Pazy. Semigroups of linear operators and applications to partial differential equations, volume 44. Springer New York, 1983.

[42] J. Printems. On the discretization in time of parabolic stochastic partial differential equations. ESAIM: Mathematical Modelling and Numerical Analysis, 35(6):1055-1078, 2001.

[43] R. Qi and X. Wang. An accelerated exponential time integrator for semi-linear stochastic strongly damped wave equation with additive noise. Journal of Mathematical Analysis and Applications, 447(2):988-1008, 2017.

[44] R. Qi and X. Wang. Optimal error estimates of Galerkin finite element methods for stochastic Allen-Cahn equation with additive noise. Journal of Scientific Computing, 80(2):1171-1194, 2019.

[45] M. Sauer and W. Stannat. Lattice approximation for stochastic reaction diffusion equations with one-sided lipschitz condition. Mathematics of Computation, 84(292):743-766, 2015.

[46] V. Thomée. Galerkin finite element methods for parabolic problems. Springer-Verlag, 2006.

[47] M. V. Tretyakov and Z. Zhang. A fundamental mean-square convergence theorem for SDEs with locally Lipschitz coefficients and its applications. SIAM J. Numer. Anal., 51(6):3135-3162, 2013.

[48] X. Wang and S. Gan. The tamed Milstein method for commutative stochastic differential equations with non-globally Lipschitz continuous coefficients. Journal of Difference Equations and Applications, 19(3):466-490, 2013.

[49] X. Wang, S. Gan, and J. Tang. Higher order strong approximations of semilinear stochastic wave equation with additive space-time white noise. SIAM Journal on Scientific Computing, 36(6):A2611-A2632, 2014.

[50] X. Wang and R. Qi. A note on an accelerated exponential euler method for parabolic spdes with additive noise. Applied Mathematics Letters, 46:31-37, 2015. 